\title{
Sequence-Level Reference Frames In Video Coding
}

\author{
Mohammad Jubran, Alhabib Abbas, and Yiannis Andreopoulos, Senior Member, IEEE
}

\begin{abstract}
The proliferation of low-cost DRAM chipsets now begins to allow for the consideration of substantially-increased decoded picture buffers in advanced video coding standards such as HEVC, VVC, and Google VP9. At the same time, the increasing demand for rapid scene changes and multiple scene repetitions in entertainment or broadcast content indicates that extending the frame referencing interval to tens of minutes or even the entire video sequence may offer coding gains, as long as one is able to identify frame similarity in a computationally- and memory-efficient manner. Motivated by these observations, we propose a "stitching" method that defines a reference buffer and a reference frame selection algorithm. Our proposal extends the referencing interval of inter-frame video coding to the entire length of video sequences. Our reference frame selection algorithm uses well-established feature descriptor methods that describe frame structural elements in a compact and semantically-rich manner. We propose to combine such compact descriptors with a similarity scoring mechanism in order to select the frames to be "stitched" to reference picture buffers of advanced inter-frame encoders like HEVC, VVC, and VP9 without breaking standard compliance. Our evaluation on synthetic and real-world video sequences with the HEVC and VVC reference encoders shows that our method offers significant rate gains, with complexity and memory requirements that remain manageable for practical encoders and decoders.
\end{abstract}

\section{INTRODUCTION}

It is well known for more than 20 years that video compression is assisted by increasing the number of reference frames used in inter-frame prediction [1], [2]. This has led all current video coding standards to support the use of multiple reference frames (MRF) for motion compensated prediction [1], [2]. However, increased MRF support is known to bring rate savings only up to a point, above which no rate gains can be obtained as there is too much redundancy within the stored frames to offer better prediction information for the increased MRF indexing cost [3]-[5]. This is why several authors [3]-[5] show that selecting the MRF option in HEVC [6] is most useful when encoding videos that comprise scenes with repetitive motion and pixel displacements.

The topic of MRF is now of renewed interest due to the compounded effect of two independent trends. The first is the continuing manifestation of significant price drops of dynamic random access memory (DRAM) chipsets ${ }^{1}$. Beyond their lower price, new DRAM designs also have decreased power consumption and decreased latency, which allows for increasing the temporal scope of frame referencing to further

MJ is with the Dept. of Electrical \& Computer Engineering, Birzeit University, West Bank, Palestine (email: mjubran@birzeit.edu). AA and YA are with the Electronic and Electrical Engineering Department, University College London, London, U.K., \{alhabib.abbas, i.andreopoulos@ucl.ac.uk). AA and YA were supported by the UK EPSRC, grant EP/R025290/1.

${ }^{1}$ see "God DRAM, that's a big price drop: Memory down 30 per cent, claim industry watchers" (The Register, Mar. 7, 2019) and https://www.dramexchange.com/ improve coding efficiency at modest increase of decoding time and energy consumption. The second trend is the increasing frequency of shot repetition within entertainment and broadcast content, in conjunction with the decreasing shot length [7]-[10]. Indeed, it has been observed that multiple nearly-identical short-length shots tend to appear in interleaved manner across medium and long time intervals in order to keep the audience engaged and assist visual memory (e.g., in talk shows or narration [7]).

Motivated by these two trends, we propose a frame inspection method that extends the referencing scope of MRF methods to the whole length of video sequences, which gives an extended set of sequence-level reference frames. To do so in a feasible manner, we use hand-crafted and neural-network based compact image descriptors for the semantic analysis of image content [11]-[13], to derive global prediction references without exhausting the memory resources of modern playback devices. The main contributions of our work are:

1) We propose feature-based semantic content analysis methods for sequence-level reference selection in block motion estimation of advanced video coding standards.

2) We propose an algorithm (Algorithm 1) for selecting frames that can be used for prediction globally, i.e., across the entire length of a video sequence; the algorithm leverages the selection process according to frame "repetitiveness" and "similarity" measures, in order to select the most beneficial (i.e., most repetitive) frames to add to reference picture sets that are also not overlysimilar to each other.

3) We evaluate our proposed method using both handcrafted and neural-network based compact descriptors, and illustrate the conditions under which our proposal allows for significant coding gains. Our benchmarks encompass the High-Efficiency Video Coding (HEVC) standard of MPEG/ITU-T, the AOMedia VP9 standard, and the recently-announced Versatile Video Coding (VVC) standard of MPEG/ITU-T.

The remainder of this paper is organized as follows: In Section II, we review recent literature related to our work and motivate the benefits of including advanced long-term referencing methods in video coding. Section III details how sequence-level referencing operates in conjunction with reference picture sets of HEVC and VP9. Section IV presents the proposed semantic similarity matching for the selection of reference frames. Section V evaluates the coding efficiency of our sequence-level referencing approach within the HEVC and VVC standards. Finally, Section VI concludes the paper.

\section{RELATED WORK}

Several techniques have been proposed in order to maximize the rate saving and efficiency offered by multiple frame 
referencing in block-based motion estimation [3]-[5], [14][17]. To reduce the memory footprint of MRF, Huang et al. [14] explore correlations between successive frames to limit the number of reference pictures used for each frame. Shen et al. [15] proposed to select MRF frames by inspecting correlations among neighboring blocks of pictures and homogeneities among areas local to individual objects. Su and Sun [18] investigated correlations between motion vectors across different frames in order to achieve fast MRF motion estimation with minimal sacrifice in coding efficiency. To mitigate unnecessary complexity overheads, other work [19], [20] studies methods by which to accelerate the selection of reference frames in MRF contexts. Frame prediction via longterm reference pictures was originally introduced by Weigand et al. [1]; short term reference (STR) pictures and long term reference (LTR) pictures are jointly considered for motion estimation. Specifically, in their work and subsequent work [21]-[26], STR pictures are frames that occur immediately before the frame being processed, while LTR pictures are previously decoded frames which are used to reference still and background regions. Similar methods [3], [4] use the PSNR of frames that follow LTR frames to determine the quality of the next LTR frame. In the work of Liu et al. [5], a jump parameter is updated for every encoded frame to give the chance for every frame to serve as an LTR. The selection of such frames is optimized in a rate-distortion (RD) sense.

The MRF aspects of the HEVC [27], [28] and VP9 [29] effectively culminated from the selection of the best of such ideas for inclusion into these standards. The VP9 standard takes MRF support further via the use of summarised frame references (a.k.a., "golden" frames), which are no-show frames used for the sole purpose of inter-frame prediction. Summarised frames are constructed by cropping and combining selected groups of frames that have frequently occurring textures. For example, VP9 [29] supports three types of reference frames: predictors from the immediate past dubbed as "last" references, summarised predictors constructed from past frames (i.e., the far and near past) denoted as "golden" frames, and "altref" frames which are constructed from distant future frames through temporal filtering. In related work [21][26], golden frames are used for background referencing, and are updated based on video content such as speed of motion and other coding statistics, and some of these works use golden frames to propose low-delay encoders. For example, Ding et al. [22] model background pictures from original video frames to be used as a part of the motion estimation in order to improve coding efficiency. To draw more coding efficiency from MRF prediction, Lin et al. [30] proposed to extend the number of reference frames for forward and backward prediction. Other work [31] proposed to add a second "altref" frame in order to balance forward and backward referencing and to provide better RD-optimization for choices of interprediction frames.

In more recent work, summarised golden frames [30], [31], are derived from groups of successive frames within a limited period. To avoid unnecessary signaling when using golden frames, recent work [32] proposed to adaptively decide golden frame coding structures by inspecting stillness in video sequences and proposed the use of multi-layer golden frame coding structures for frame groups that are not static across successive frames. Other proposals use golden frames in hierarchical bi-predictive picture (HBP) structures [24], where I-frames are coded at the beginning of scenes and used as references augmented to frames normally used for prediction in HEVC. Frames are added in reference picture sets (RPS) of HEVC in such a way represent the ground truth background of a scene, and Gaussian mixture models are used to determine the most suitable frames to use as background references [24]. Zuo and $\mathrm{Yu}$ [33] assign specific scene-cut frames as LTRs for the coding of GOPs. These scene-cut frames are encoded with lower QPs to ensure fidelity. To select LTRs, motionfield distance kernels are used to determine an appropriate demarkation of K-means clusters comprised entirely of scenecut frames [33]. A subset of cluster centers is then assigned as the set of LTRs to be used during encoding, where the subset is determined by optimizing an objective on: (i) the total number of bits to encode all cluster centers (i.e., all LTRs), and (ii) the total distance between all scene cut frames and their respective cluster centers (i.e., correlating with the girth of clusters). Chen et al. [25] proposed block-composed background references (BCBR) for long term prediction, where pixel blocks in the reference are updated gradually to replace foreground content and hence update the LTR, and recent work by Ma et al. [26] considers cropped regions selected from reconstructed frames. In their work, frames are maintained in the DPB if their repetitiveness exceeds a predefined threshold. However, it is well known that sprite generation is inaccurate when scenes contain fast camera motion artifacts, which is why background reference generation techniques used in related efforts [21][26] usually need videos with minimal global motion in order to provide coding gains. In these efforts, commonality within groups of pictures is considered to construct long term references, which makes their use suitable for videos where repetitiveness can be estimated locally, or within short periods of playtime.

Coupled with the capabilities and efficiency of current DRAM hardware, our work is motivated by the above efforts which show that, when reference frames are selected with prior knowledge of the content to be encoded, long-term referencing across wider time intervals can improve coding efficiency. Unlike previous work that selects reference frames via complex pixel-level error distance criteria that are, by necessity, limited to short time intervals, we are the first to propose the use of compact feature descriptor methods [11][13] to determine the repetitiveness and semantic similarity between frames at very long time scales for MRF in video coding. This allows for the extension of the MRF concept to the limit case of sequence-level referencing. In order to demonstrate that this can be implemented within existing standards, and to overcome the "short-sighted" nature of their prediction picture sets (which restrict the inter-frame predictors to local temporal proximities), we augment reference picture sets of the HEVC [27] and VVC [34] reference software with sequence-level frames and validate our proposal with videos comprising synthetic and naturally-occurring repetition of scene semantics. 


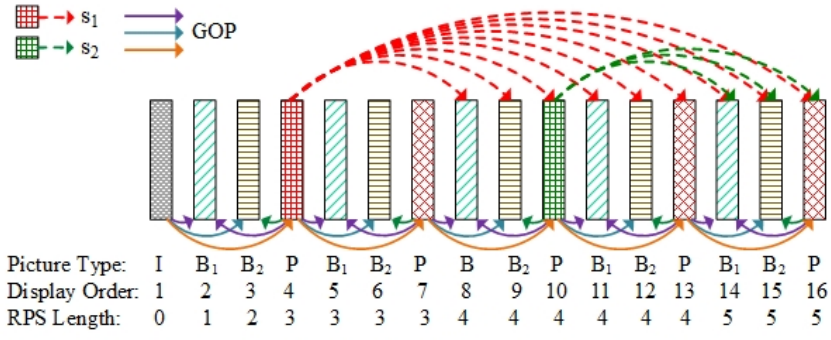

Fig. 1: Example of how stitching frames in $\mathcal{S}$ are used for interprediction across GOPs. Shading patterns signify frame types used in prediction; solid lines (shown underneath the frames) indicate standard GOP inter-frame prediction direction; dashed lines (shown above the frames) highlight inter-prediction direction using stitching frames selected by our proposal and inserted into the RPS.

\section{Extended Long-Term Prediction}

We first introduce sequence-level frame referencing structures and illustrate how existing video codecs can support such structures without breaking the operation of a standard decoder (Section III-A). We then move on to describe the operation of the proposed algorithm for sequence-level reference selection.

\section{A. Defining Prediction Structures}

We describe how we can modify the HEVC reference software [27] to enable the use of frames over the entire length of a video sequence as inter-frame predictors. In HEVC, the RPS specify which frames are used for inter-frame prediction, and decoded picture buffers (DPB) store reconstructed frames to be used for future prediction. As long as an HEVCcompliant decoder maintains the equivalent RPS and DPB, the encoded bitstream can be decoded without modifications to the standard. Because the DRAM available on playback devices is finite, the size of allocated DPB memory is the dominant factor limiting the temporal distance between frames and their corresponding predictors that can be used for interframe prediction.

In our proposal, we consider a set of video frames $\mathcal{F}=$ $\left\{f_{1}, f_{2}, \ldots, f_{M}\right\}$ from which we determine the set of stitching frames $\mathcal{S}=\left\{s_{1}, s_{2}, \ldots, s_{N}\right\}$ to be used as extended longterm reference pictures in HEVC (and detail the processes of determining $\mathcal{S}$ in Section III-B). That is, frames in $\mathcal{S}$ are retained in the DPB upon being decoded, and are specified as inter-frame predictors in the RPS of all subsequent frames. Fig. 1 illustrates how frames in $\mathcal{S}$ are used for the prediction of future frames within a standard group-of-pictures (GOP) prediction structure: the RPS length increases permanently when frames with display order 4 and 10 are added as stitching frames $s_{1}$ and $s_{2}$ during the encoding of display-order frames 8 and 14. These two additional stitching frames are linked to GOP inter-frame prediction via the dashed arrows shown at the top part of Fig. 1, and are already available to the decoder as they have already been encoded via the GOP prediction structure.

Importantly, when including sequence-level references we do not modify underlying GOP structures (which can include both STR and LTR frames), and only augment the RPS of subsequent frames to include more frames for inter-prediction. This is useful when considering different GOP profiles that use multiple reference frames [6] (e.g., extended long-term references in $\mathcal{S}$ can be used without interfering with GOP structures when hierarchical or bi-directional prediction is used). Aggregating sequence-level references increases the RPS size monotonically as the Picture Order Count (POC) of frames increases (as shown in Fig. 1). To accommodate this, we modify the memory allocated for the DPB to allow for $N$ stitching frames in addition to the maximum number of frames used for inter-frame prediction in the specified GOP structure.

The proposed modifications can be easily implemented in the HM reference software of the HEVC standard [27]. Specifically, this can be achieved using the POC of frames (which in $\mathrm{HM}$ is defined as a control variable picture_count, and is equal by definition to $M$ ), and explicitly define the RPS of all frames by modifying the control variables reference_pictures, deltaRPS, and reference_idcs. To reduce the signaling overhead associated with the increased length of RPS due to the inclusion of extended long-term references, the inter-RPS option [27] can be used encode the Sequence Parameter Set (SPS) of all frames. Similarly, for the VP9 reference software [28], this can be achieved by modifying vp9_encoder and vp9_ratectrl to allow for the explicit definition of frames used for inter-frame prediction, and setting the parameter baseline_gf_interval to allow for the required GOP size needed for sequence-level frame referencing.

\section{B. Selecting Sequence-level "Stitching" Frames}

We present the frame selection process for sequence-level referencing. Our approach leverages on the semantic similarity between frames as measured by descriptor based matching methods ${ }^{2}$ such as ORB or VLAD [11], [12], or as predicted by independently fitted state-of-the-art models such as convolutional neural networks (CNNs) [13], [35] and imagetranslation variants thereof [36], [37]. To take advantage of the low-complexity nature of ORB and similar methods, we formalize our notation with respect to descriptor matching methods, and later highlight the relevance of CNNs and the extent to which they can replace descriptor matching in selecting long-term reference frames. In order to provide for the best prediction efficiency, frames included in $\mathcal{S}$ must capture long-term correlations between scenes while providing the least amount of redundancy in RPS. To determine which frames are included in $\mathcal{S}$, as a first criterion of selection we measure the repetitiveness within a video segment, $r_{m}$, of semantic elements in $f_{m}$. To do so, we use a mapping $f \mapsto d(f)$ to map frames onto a compact descriptor space, and define the semantic likeness $l$ between a frame $f_{m}$ and some set of frames $\mathcal{X}$ as the inverse of average distance between $f_{m}$ and all frames in $\mathcal{X}$ :

\footnotetext{
${ }^{2}$ In Section IV we summarize the utilized methods and empirically validate the correlation between semantic similarity of content and the rate efficiency of block motion estimators.
} 


$$
l\left(f_{m}, \mathcal{X}, K\right)=\left(\frac{1}{K} \sum_{f_{i} \in \mathcal{X}} \phi\left(d\left(f_{m}\right), d\left(f_{i}\right)\right)\right)^{-1}
$$

where $K$ is the number of frames in $\mathcal{X}$ and $\phi\left(d\left(f_{m}\right), d\left(f_{i}\right)\right)$ is a function that measures the distance between calculated compact descriptors of frame $f_{m}$ and frame $f_{i}$ (e.g., as the brute force matching distance of ORB features [12], or the inner product of VLAD vectors [11], or the inner product of CNN-based compact feature descriptors [36]-[38]). The distance term $\phi$ can accommodate for any image matching method, where $d\left(f_{m}\right)$ and $d\left(f_{i}\right)$ can denote ORB feature vectors [12], or in the case of state-of-the-art CNN models, $d\left(f_{m}\right)$ and $d\left(f_{i}\right)$ can denote the compacted mappings of last-layers [36]-[38]. In Section IV we further discuss how $\phi\left(d\left(f_{m}\right), d\left(f_{i}\right)\right)$ measures the distance between descriptors in both cases. Note that descriptor distance in (1) is inversely proportional to frame similarity and that $\phi$ considers distance in compact descriptor space rather than the pixel-domain. The latter is important as, when our approach scales to sequencelevel referencing, we only need to retain compact descriptors in memory, which for ORB typically comprise a few hundred bytes per frame [11], [12], [39]. This can also be tuned for CNNs by adjusting the hyperparameters pertaining to their last-layer dimensions [36]-[38] thus making referencing and subsequent computation of $\phi$ feasible.

From (1), we quantify the semantic repetitiveness of frame $f_{m}$ in $\mathcal{F}$ as $r_{m}=l\left(f_{m}, \mathcal{F}, M\right)$, and initialize the stitching frame set $\mathcal{S}$ by setting $s_{1}$ to be the frame $f_{m}$ associated with the highest value of $r_{m}$. Note that videos can be preprocessed to measure $\phi\left(d\left(f_{m}\right), d\left(f_{i}\right)\right)$ of (1) in an independent pass that precedes video coding, and before selecting sequence-level references. If streaming video-on-demand $(\mathrm{VoD})$ content, this pass can encompass the entire video sequence. If streaming live video content, the pass to measure $\phi\left(d\left(f_{m}\right), d\left(f_{i}\right)\right)$ of (1) is merged with the actual video encoding and only past frames up to the current frame can be used. To enforce a wider coverage of spatial features and more variance in the content of $\mathcal{S}$, stitching frames subsequent to $s_{1}$ are selected by considering $r_{m}$ and a second criterion of selection $u_{m}$ which measures the uniqueness of $f_{m}$ among frames previously selected in $\mathcal{S}$. Specifically, for the $i^{t h}$ stitch frame selection we denote previously selected stitching frames by $S^{i}=\left\{s_{1}, \ldots, s_{i-1}\right\}$, and, via (1), we calculate $u_{m}^{i}=l\left(f_{m}, \mathcal{S}^{i}, i-1\right)^{-1}$ as the second frame selection criterion. Note that $u_{m}^{i}$ is calculated as the inverse of (1), to give higher selection priority to candidate stitch frames that are semantically dissimilar to the ones already available in $S^{i}$.

To quantify how a candidate stitch frame $f_{m}$ is to be the next frame included in $\mathcal{S}^{i}$, we define the "next stitch candidate" criterion $c_{m}^{i}$ as the linear combination of $r_{m}$ and $u_{m}^{i}$ :

$$
c_{m}^{i}=w_{r} r_{m}+w_{u} u_{m}^{i}
$$

where $w_{r}$ and $w_{d}$ are the weights used to scale the importance of repetitiveness of frames in $\mathcal{F}$ and their uniqueness among frames in $\mathcal{S}^{i}$, respectively. Because both $r_{m}$ and $u_{m}^{i}$ are calculated from (1) and its inverse, a linear combination can determine the best counterbalance of importance versus uniqueness of each candidate stitch frame. This is validated experimentally in Section V.

For every iteration $i$, frames associated with the highest value of $c_{m}^{i}$ are appended to $\mathcal{S}^{i}$ to make it into $\mathcal{S}^{i+1}$, until $i=N$ and the full stitching set $\mathcal{S}$ is determined. In Fig. 4 we show an example of how the selection criterion $c_{m}^{i}$ changes with every iteration. As shown in the figure, every position $m$ that maximizes $c_{m}^{i}$ becomes a local minimum for $c_{m}^{i+1}$, since the frame's uniqueness measure $u_{m}$ is minimized after it has been included in the set of stitching frames $\mathcal{S}$. This ensures this frame, as well as similar frames to it, are not included as further stitch frames. As a result, this enforces a wider coverage of spatial features and more variance in the content of $\mathcal{S}$. This selection process of frames for $\mathcal{S}$ is formally presented in Algorithm 1. The entire process can run as a separate pass prior to encoding, and, as shown in Fig. 1, the selected frames are inserted in $\mathcal{S}$ after they have been encoded, so as for them to be available to the decoder.

\section{Closing Remarks on Algorithm 1}

Balancing between semantic similarity and uniqueness: Frames that have already been included in $\mathcal{S}$ are implicitly ignored in subsequent sequence-level reference selection steps, since the term $u_{m}^{i}$ in Algorithm 1 accounts for how unique each candidate is to frames in the set $\mathcal{S}$. That is, if a frame is already included in $\mathcal{S}$, its respective criterion of selection $c_{m}^{i}$ will be relatively low (as can be seen from the square indicators indicating dips in Fig. 4), and is therefore not selected to be included in $\mathcal{S}$ again. Moreover, if a short scene does not repeat very often, the probability of a frame being selected from it as a sequence-level reference is also low. This is because frames from scenes that are semantically isolated from the rest of the video inherently produce a low value for $w_{r} r_{m}$ in (2), which quantifies how often the semantics of a candidate frame occur across the video.

Application in VoD and live streaming: While our work is more suitable for VoD streaming (where compression happens offline, prior to transmission), Algorithm 1 can also be adapted to live streaming by not allowing the selection of future frames to be included in the set $\mathcal{S}$. In this work, we focus on $\mathrm{VoD}$ streaming and validate our method for long sequences and entire-sequence encoding, but future work can also validate our method for live encoding, where numerous other issues like encoding and decoding delay must be taken under careful consideration.

Complexity: As presented, Algorithm 1 requires $O\left(N M^{2}\right)$ operations. However, the computations of semantic likeness $l\left(f_{m}, \mathcal{S}^{i}, i-1\right)^{-1}$ of line 8 do not need to be repeated for each inner for-loop. By calculating a moving average of $u_{m}^{i}$ instead, redundant computations of $l\left(f_{m}, \mathcal{S}^{i}, i-1\right)^{-1}$ can be avoided to reduce the complexity of the second loop (see lines 4-9) in Algorithm 1 to $O(N M)$. Beyond this, for the utilized descriptors and distance function, the distance function of (1) is symmetrical, i.e., $\phi\left(d\left(f_{m}\right), d\left(f_{i}\right)\right)=\phi\left(d\left(f_{i}\right), d\left(f_{m}\right)\right)$. That means that distances between pairs of frames need to 
be calculated only once. Exploiting this, we can halve the complexity of the first loop in Algorithm 1 (see lines 1-2) which is $O\left(M^{2}\right)$ complex. Finally, in Section $\mathrm{V}$ we show how local temporal-coherence in video scenes can be exploited to further reduce the time complexity of Algorithm 1.

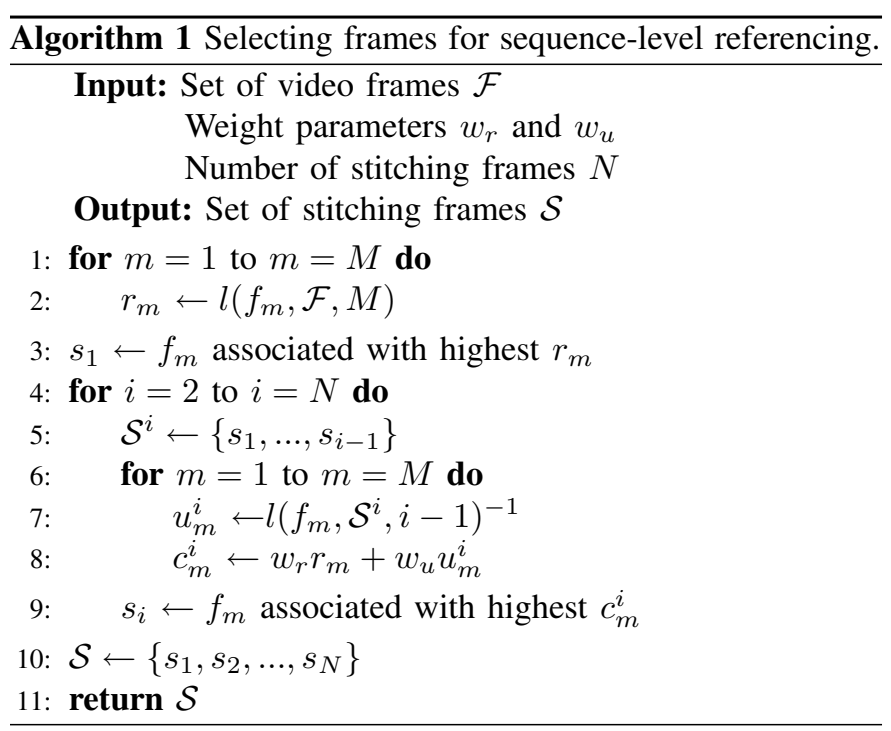

\section{Cross-Frame Semantic Matching}

Since our approach hinges on semantic similarity between frames measured via compact descriptors, we summarize recent techniques for such descriptors and demonstrate how their similarity correlates with the rate efficiency of inter-frame prediction in HEVC.

Efficient semantic similarity matching techniques use specially-crafted descriptors (represented in fixed-length vectors) to describe local regions of salient visual features, and define low-complexity functions to measure a notion of distance between different descriptors. Under this class of techniques, ORB descriptors [12] and VLAD [11] are commonly used for high level semantic description of images [40], [41], and can be independently used as processing blocks in systems for measuring the semantic similarity between frames [42]. When encoding complexity is not constrained, complex mapping methods can be used to learn the expected coding efficiency of video codecs directly from image pairs. For example, the latter can be achieved by state-of-the-art CNN architectures [13], [36], [37] which were shown to yield the best performance on pair-based computer vision tasks (e.g., to match and identify landmarks or human faces). However, CNNs and their derivatives tend to require significantly more operations than hand-crafted descriptors, and may be unnecessary in situations where tunable low-complexity matching methods can suffice.

Low-complexity counterparts to CNNs include ORB feature matching methods, which comprise histogram vectors for local regions of frames to describe the frequency of occurrence of luminance gradients that move in different directions (commonly 8 directions are used, to describe luminance gradients moving up, up-right, right, and so on). In doing so, ORB features give a compact description of salient regions in frames

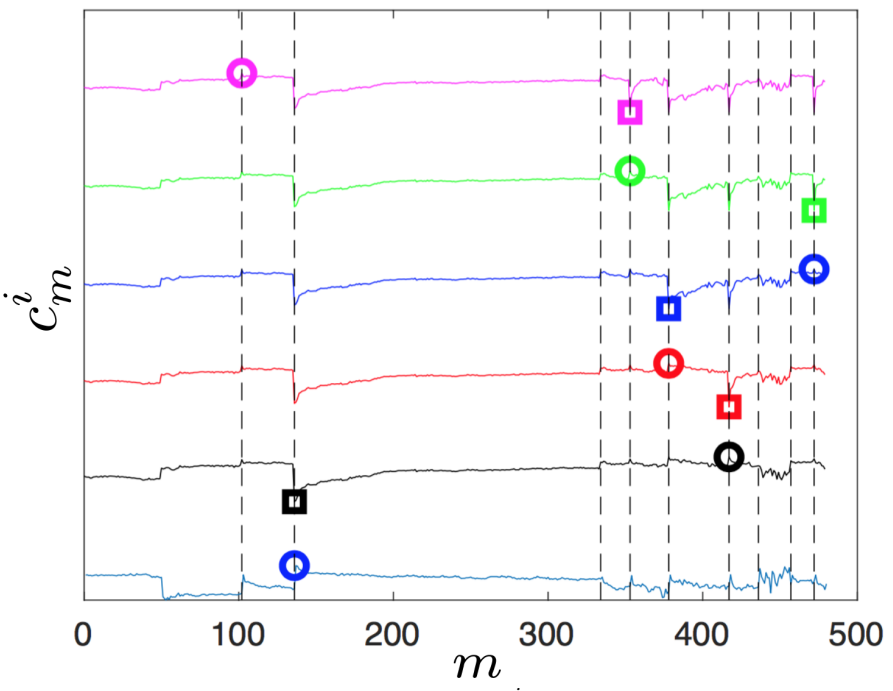

Fig. 4: Example plots of $\forall m: c_{m}^{i}$ with $i$ increasing from bottom to top. Circles indicate selected frames to be included in $\mathcal{S}^{i+1}$ and squares show frames last included in $\mathcal{S}^{i}$.

as fixed-length vectors, and different frames give different numbers of vectors subject to the the number of salient regions within each frame [12].

Because the coding efficiency of motion estimation is highly correlated with how similar predicted frames are to their predictors, we expect that the coding efficiency should inherently correlate with: (i) the matching distance between visual features of frames as described by hand-crafted ORB descriptors [12], [42], and (ii) CNNs trained to map image pairs to coding efficiencies of prescribed video codecs via their inter-frame prediction models. To demonstrate this, in Fig. 2 we consider a single target frame $f_{t}$ from a test video and show the compact descriptor distance measured prior to sequencelevel reference selection, $\phi\left(d\left(f_{t}\right), d\left(f_{m}\right)\right)$, and the required rate for inter-frame prediction, $r\left(f_{t}, f_{m}\right)$, when predicting $f_{t}$ from $f_{m}$ for all frames in $\mathcal{F}$.

When calculating $\phi\left(d\left(f_{t}\right), d\left(f_{m}\right)\right)$ as the ORB distance between any frame pair $\left(f_{t}, f_{m}\right)$, we follow the standard practice [12], [39] of measuring the average of all distances between the constituent descriptors of $\left(f_{t}, f_{m}\right)$, where each descriptor in $f_{t}$ is matched against the descriptor in $f_{m}$ closest to it in euclidean feature space. On the other hand, in measuring distances $\phi\left(d\left(f_{t}\right), d\left(f_{m}\right)\right)$ via CNNs, similar to previous work on semantic matching in other domains [36]-[38], we train a model to return a learned distance metric for any input pair of frames $\left(f_{t}, f_{m}\right)$, where the inter-prediction rate of any given codec $r\left(f_{t}, f_{m}\right)$ is used as the objective ground-truth during training. Specifically, and to study more complex variants of rate predictors, we train the $\mathrm{CNN}$ architecture of [36] on a subset of Kinetics-400 [43] videos to translate frame pairs to the rate required by HEVC to encode $f_{t}$ from $f_{m}$. To train this rate prediction model, we first encode Kinetics-400 videos via HEVC as a preprocessing step. The CNN is then trained with tuples $\left(f_{t}, f_{m}, r_{t}\right)$, where it maps a concatenated volume of two frames $f_{t}$ and $f_{m}$ to the ground truth coding rate $r_{t}$ required by the pre-specified video codec (i.e., HEVC) to predict $f_{t}$ from $f_{m}$. The rate prediction model was trained for 100 epochs with stochastic gradient descent [44], and a learning rate of 


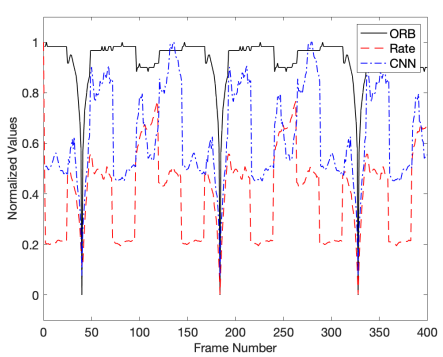

(a) $t=40$

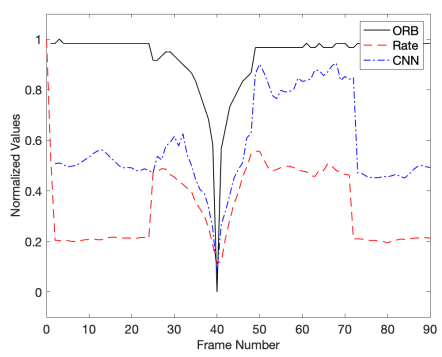

(b) Zoom in for $t=40$

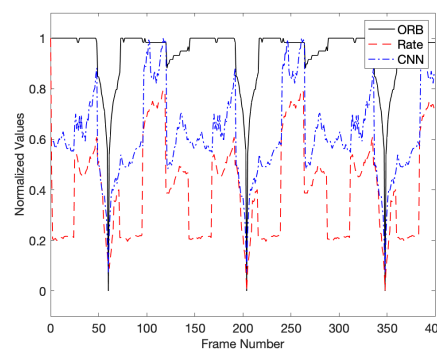

(c) $t=60$

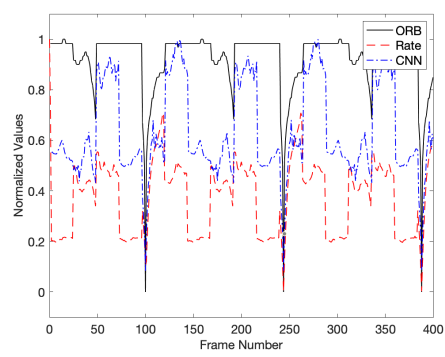

(d) $t=100$

Fig. 2: Correlation between the required rate for inter-frame prediction $r\left(f_{t}, f_{m}\right)$ and the distance $\phi\left(d\left(f_{t}\right), d\left(f_{m}\right)\right)$ as measured by normalized values of ORB matching distance, and frame coding rates as predicted by an independently trained CNN. This is shown for target indices $t \in\{40,60,100\}$ and predictor indices $m \in[1,400]$.

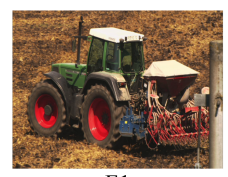

F1

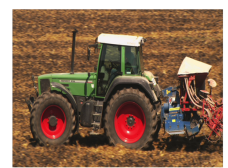

F385

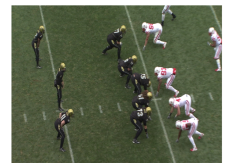

S1 (F441)

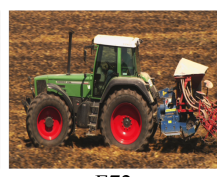

F72

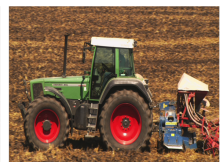

F432

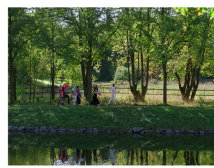

S2 (F582)

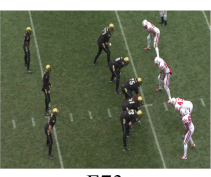

F73

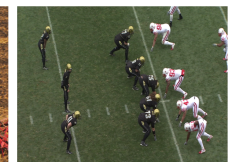

F433

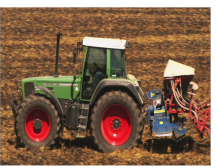

S3 (F404)

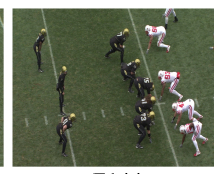

F144

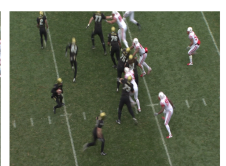

F504

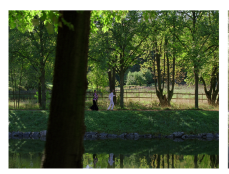

S4 (F305)

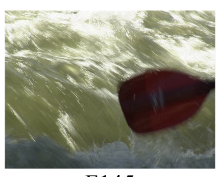

F 145

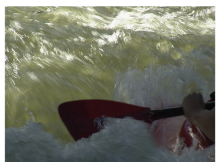

F505

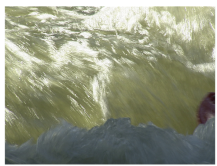

S5 (F195)

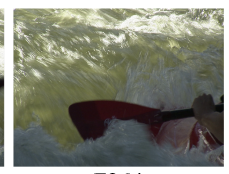

F264

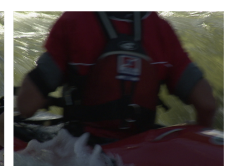

F576

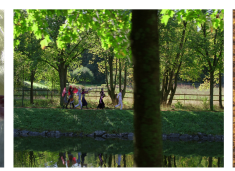

S6 (F672)

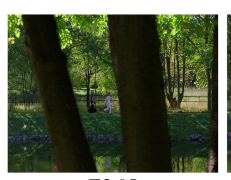

F265

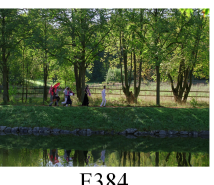

F384

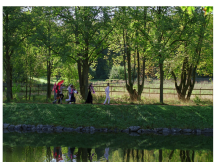

F577

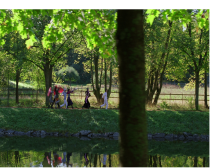

F672

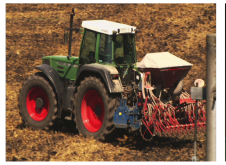

S7 (F4)

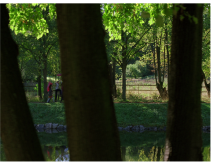

S8 (F274)

Fig. 3: A video example of frames included in $\mathcal{S}$ when $N=8$ and $M=672$. By considering the two parameters $r_{m}$ and $u_{m}^{i}$ using the ORB distance function $\phi\left(d\left(f_{t}\right), d\left(f_{m}\right)\right)$, frames are included in $\mathcal{S}$ to be used as predictors for all subsequent frames in the decoding order. The first two rows show the video sequence with " $F$ " indicating frame order, and the last row shows frames selected as sequence-level references with " $\mathrm{S}$ " indicating the order of inclusion in $\mathcal{S}$.

\section{$2 \times 10^{-5}$.}

From a test sequence comprising 400 frames, in Figure 2 we show indicative comparisons of $\phi\left(d\left(f_{t}\right), d\left(f_{m}\right)\right)$ against $r\left(f_{t}, f_{m}\right)$ when $t \in\{40,60,80,100\}$. For clear presentation, we show normalized values of $r\left(f_{t}, f_{m}\right)$ and $\phi\left(d\left(f_{t}\right), d\left(f_{m}\right)\right)$ : lower values of $\phi\left(d\left(f_{t}\right), d\left(f_{m}\right)\right)$ indicate closer semantics between frames, and the distance is 1 when no semantic correspondence exists between salient local regions in $f_{t}$ and $f_{m}$. From Fig. 2 we see that, while $\phi\left(d\left(f_{t}\right), d\left(f_{m}\right)\right)$ is 1 for most frames when $f_{t}$ is not in the neighborhood of $f_{m}$, $r\left(f_{t}, f_{m}\right)$ and $\phi\left(d\left(f_{t}\right), d\left(f_{m}\right)\right)$ are highly correlated when $f_{t}$ and $f_{m}$ are within close temporal proximity. However, this correlation is not always existent (e.g., when $t=40$ and $m \in[50,80]$ in (d) of Fig. 2), this is because some frames are not visually complex, and intra-frame prediction is sufficient for encoding such frames without much need for the use of inter-frame prediction (e.g., black screens or frames with static backgrounds). In Fig. 3 we show frames selected as sequence-level stitch references by using Algorithm 1 and considering ORB distance as the semantic distance function $\phi\left(d\left(f_{t}\right), d\left(f_{m}\right)\right)$. Overall, we also observe that the prediction of the trained $\mathrm{CNN}$ correlates better with the rates required by the codec. However, similar to ORB and due to variable modes of prediction, the $\mathrm{CNN}$ predicts rates less reliably when the indices of $f_{m}$ and $f_{t}$ are further apart from each other.

Finally, and with regards to the complexity of determining semantic similarities between pairs of frames, the trade-off between complexity and reliable prediction of CNN methods has to be tuned via sophisticated techniques which include static and active weight pruning [45]-[47]. In contrast, the complexity of matching ORB descriptors [12] is directly related to the number of dimensions of feature descriptors $D$, and is therefore directly adjustable. Specifically, matching a reference frame with $k_{m}$ detected features against a target frame with $k_{t}$ detected features requires $k_{t} \times k_{m} \times D$ floating point operations. Since the complexity overhead of frame matching is directly related to the number of detected features $k_{t}$ and $k_{m}$, the complexity of detecting sequence-level frame references can be reduced by increasing thresholds of feature detection (thereby reducing $k_{t}$ and $k_{m}$ ). In this way, selecting sequence-level reference frames can be tuned to accommodate for any restrictions on complexity when encoding. We finally note that, due to the modest and tunable aspects of its complexity, ORB feature matching is more suitable for typical 
video coding contexts that have constraints on complexity. The efficacy of ORB for practical deployment is compounded by the fact that it performs comparably to CNNs when predicting coding rates of standard codecs. Hence, in Section $\mathrm{V}$ we validate the performance of both methods on standard video coding datasets, with a focus on ORB feature matching for the selection of sequence-level reference frames.

\section{EXPERIMENTAL RESULTS}

To quantify the coding gains achievable by our method, we evaluate on two content types (see Table I):

1) Synthesized Videos: we use videos from the XIPH [48] and UVG [49] repositories to synthesize test sequences and control the amount repeatability across scenes of videos. In this way we show the conditions in video content that make the use of long-term referencing more beneficial to coding efficiency.

2) Natural Videos: we use videos from the FILMshort [50] and UVG [49] public repositories to validate our method on videos with naturally-occurring scene repetition and scene progression.

We compare and contrast the use of extended sequencelevel references in HEVC against three benchmark coding methods: (i) the reference HEVC software [27] with only STR frames enabled ${ }^{3}$, (ii) VP9 [28] when using summarised "golden frames" to store recurrent texture in video (which can be seen as an alternative to our proposed sequence-level stitch frames), and (iii) an HEVC implementation that selects as LTRs the GOP-LTP frames of Zhuo and Yu [33], which is closest to ours in terms of usage of LTR. To enable the use of extended sequence-level "stitching" references for interframe prediction in HEVC via our method, we modified the HM reference software [27] as described in Section III-A, and hereafter we refer to this instantiation of our method as HMS, abbreviating HEVC with Multiple Stitching frames. Since HEVC is more ubiquitous compared to VP9 and VVC [51], we provide our exhaustive study focusing on HEVC, and report indicative results for the less common codecs like VP9 and VVC. For all codecs, we encode videos to various bitrates by setting the Constant Rate Factor (CRF) for HEVC and VP9, and use inter-frame prediction for all frames subsequent to the first (i.e., only the first frame is encoded as an I frame). In all tests pertaining to HEVC encoding, we use the main coding profile (level-4.1). To further understand the extent to which sequence-level referencing is of benefit to coding efficiency, we test on two GOP profiles: one using a uni-directional IPPP coding structure, and another using a hierarchical IPBB coding structure. Finally, in order to reduce the size of RPS signaling, we enable inter-RPS prediction in the HM options [27].

\footnotetext{
${ }^{3}$ Since standards only specify the normative decoder operation, there is no normative method for filling the LTR buffer. Thus, the LTR option in the HEVC reference software encoder (HM) does not include any reference method for LTR selection. As such, we can only set the STR option for the HEVC HM benchmark and we implemented our approach on HEVC reference software as a method for LTR selection. In addition, we also report indicative tests for the reference software of MPEG VVC [34], which represents the state-of-the-art in standards-based video coding. While our proposal can also be implemented within AOMedia standards, we leave this as future work.
}

TABLE I: Properties of test sequences used throughout our validation on HEVC HM 16.17 and VVC VTM 7.1 reference software. For the top part ("Synthesized" type), details on the utilized sequences and the way the synthesized sequences are produced are provided in Section V-A.

\begin{tabular}{cccccc}
\hline Sequence & Resolution & $M$ & Scene Repetition & Repository & Type \\
\hline SV1 & SD & 400 & Low & XIPH & Synthesized \\
SV2 & SD & 800 & High & XIPH & Synthesized \\
SV3 & SD & 800 & Medium & XIPH & Synthesized \\
SV4 & SD & 800 & Medium & XIPH & Synthesized \\
SV5 & SD & 1200 & Medium & XIPH & Synthesized \\
SV6 & SD & 2000 & High & XIPH & Synthesized \\
BSH1 & HD & 1000 & Medium & UVG & Synthesized \\
BSH2 & HD & 1600 & High & UVG & Synthesized \\
BSH3 & HD & 2000 & High & UVG & Synthesized \\
\hline Breathe & SD & 20800 & Medium & FILMShort & Natural \\
City Lights & SD & 12000 & Medium & FILMShort & Natural \\
Naughty List & SD & 9200 & Low & FILMShort & Natural \\
Brink & SD & 8600 & Medium & FILMShort & Natural \\
Jet & SD & 8900 & Low & FILMShort & Natural \\
Big Bunny & SD & 2200 & Low & XIPH & Natural \\
HoneyBee & HD & 600 & Medium & UVG & Natural \\
Bosphorus & HD & 580 & Low & UVG & Natural \\
ShakeNDry & HD & 300 & Medium & UVG & Natural \\
\hline
\end{tabular}

For $\phi\left(f_{\mathrm{m}}, f_{\mathrm{i}}\right)$, we use brute force matching distance of ORB feature descriptors [12]. The time complexity associated with determining $r_{m}$ for $M$ frames in $\mathcal{F}$ is $O\left(M^{2}\right)$. Therefore, despite the compactness of ORB descriptors, applying our approach uniformly across all video frames will be overly complex and, to a large extent, unnecessary, since the largest gain from stitch frames will occur around scene changes. Hence, we calculate $r_{m}=l\left(f_{m}, \mathcal{F}^{\prime}, M\right)$, where $\mathcal{F}^{\prime}$ comprises only frames that exist at the beginning of each scene, and we detect scenes via the scene detection tool PySceneDetect [52]. Importantly, while $\mathcal{F}^{\prime}$ comprises only scene-cut frames, candidate frames $f_{m}$ (which are eventually matched against $\mathcal{F}^{\prime}$ and conditionally included in $\mathcal{S}$ ) can exist anywhere within a video sequence. That is, long-term references included in $\mathcal{S}$ can exist anywhere within a video, and are included in specific priority orders as determined by the proposed algorithm in Section III. This is why, constraining $\mathcal{F}^{\prime}$ to scene-cut frames reduces matching complexities and leverages temporal coherence across scenes without compromising the selection pool of long-term references. We set the number of sequence-level references, $N$, to the number of video scene cuts detected. In doing so, we ensure that sequence-level references can be selected such that scenes with recurring semantics are similar with at least one frame in $\mathcal{S}$ (although, whether $\mathcal{S}$ is selected to achieve the latter is entirely determined by Algorithm 1). For all results pertaining to sequence-level referencing, and to find the optimal balance between considering the semantic repetitiveness $r_{m}$ and uniqueness $u_{m}^{i}$ of frames to be included in $\mathcal{S}$, we set $\omega_{r}=1$ and $\omega_{u}=1$ as determined via crossvalidation to be the best performing setting (i.e., by giving the repeatability and uniqueness of frames an equal measure of priority in determining their inclusion or exclusion from $\mathcal{S})$. Note that we use a search step of 0.5 over the interval $[0,2]$ in our cross validation of both $\omega_{r}$ and $\omega_{u}$. In Table I we present the high-level properties of all videos in our evaluation. Specific to the sequences SV1-SV6 and those of the FILMShort [50] repository, we resize the videos to $640 \times 480$ standard definition (SD) using FFmpeg bicubic 
interpolation [53], while high-definition videos from UVG [49] are not resized to provide results for high-definition video.

\section{A. Evaluation On Synthesized Videos}

To synthesize the interleaved test videos SV1-SV6, where each sequence holds a different degree of scene repetition, we used three videos from the public XIPH test repository [48]: "Touchdown", "Parkjoy", "Tractor", and to synthesize BSH1BSH3 we use three videos from UVG [49] : "HoneyBee", "Bosphorus", and "ShakeNDry".

We control scene repetition in the synthesized videos SV1SV6 and BSH1-BSH3 by interleaving scenes from $K$ different videos $(2 \leq K \leq 3)$. We interleave scenes by concatenating $R$ randomly cropped segments from each video and ensuring that neighboring segments within synthesized videos always come from different video sources. This ensures there are $R$ scene changes per synthesized video. By repeating this process with increasing values of $(K, R)$, we create nine synthesized videos with different levels of scene repetition. Importantly: (i) our process of random cropping of one-second segments ensures that scenes will not repeat in their entirety and any partial scene repetition may occur at any temporal distance within each synthesized sequence; (ii) we do not perform lossy coding when creating the synthesized videos; hence, the quality of synthesized videos remains identical to the $K \mathrm{SD}$ source videos used for synthesis [48].

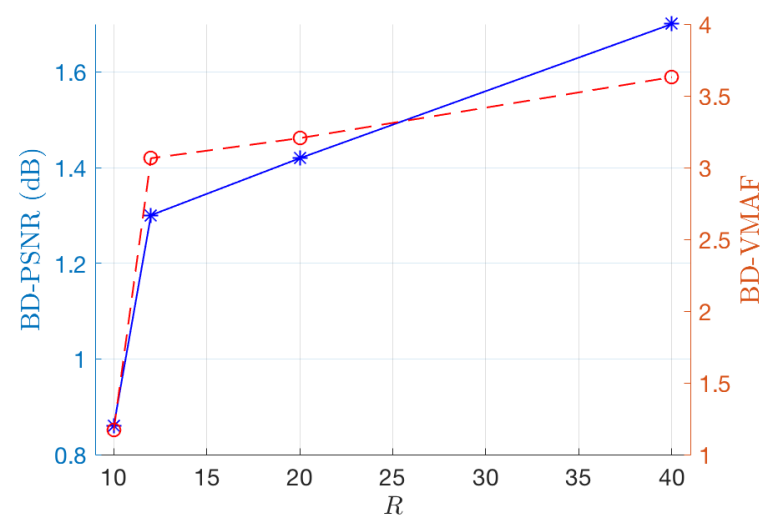

Fig. 5: Relationship between quality measured as average BDPSNR (blue, left y-axis) and BD-VMAF (red, right y-axis), and cross-scene semantic frequency measured as the number of interleaved crops extracted from each source video $R$.

Comparing our proposal HMS against the HEVC reference implementation of HM, in Table II we report the Bjontegaard Delta-rate (BD-Rate) and Bjontegaard Delta-PSNR (BDPSNR) on the luminance (Y) channel of the synthesized videos of Table I. Beyond PSNR-based evaluation (BD-Rate $\mathrm{P}_{\mathrm{P}}$ and BD-PSNR), we also report Bjontegaard delta rate and quality gains for the video multi-method assessment fusion (VMAF) metric developed by Netflix [54], [55] (presented as BD-Rate ${ }_{\mathrm{V}}$ and BD-VMAF). From Table II, when considering BD-Rate $\mathrm{P}$ results for all tested videos, we note that $34.05 \%$ and $48.07 \%$ saving is achieved when comparing HMS to the HM and VP9 anchors, respectively. In addition, BD-PSNR gains of $1.76 \mathrm{~dB}$ and $1.87 \mathrm{~dB}$ are achieved when comparing HMS to
HM and VP9, respectively. To show that sequence-level frame referencing can be applied to hybrid coding structures, we report results for $\mathrm{PBBB}$ coding structures and set the GOP size to 4 . The results in Table II show that HMS-PBBB is superior to the HM-PBBB anchor, and gives a BD-Rate $\mathrm{P}$ saving of $46.76 \%$ and a BD-PSNR gain of $2.8 \mathrm{~dB}$. In reference to VMAF, HMS and HMS-PBBB maintained an edge over the other two codecs when considering all videos. For example, HMS achieved BD-VMAF gains in the range 1.17 to 9.54 and 1.61 to 15.26 when compared to HM and VP9, respectively. The results in Table II indicate that gains increase as the lengths of the synthesized videos increase (e.g., as can be seen for SV3 and SV6). This is due to the increase in scene repetition within synthesized videos.

To better understand the conditions wherein sequence-level references are most useful, Fig. 5 shows a plot that highlights the relationship between the number of interleaved crops extracted from each source video $R$ (increased $R$ leads to increased scene and semantic repetition), and the gains achieved by HMS (shown as BD-PSNR and BD-VMAF). As expected, we see that sequence-level inter-frame prediction is indeed most useful when values of $R$ are higher (i.e., when videos are more repetitive). This agrees with the gains we observe from Table II for highly repetitive videos (also see Table I for details on scene repetition in test videos), which indicate that sequence-level references are of less benefit when scenes are short and their respective semantics do not repeat, and are of most benefit when scenes repeat frequently throughout the video.

To quantify the computation and memory usage to encode and decode videos with our proposal, we report indicative memory and relative encoding/decoding runtime requirements $\left(\mathrm{RER}=\frac{\text { Runtime(HMS Enc) }}{\text { Runtime(HM Enc) }}, \mathrm{RDR}=\frac{\text { Runtime(HMS Dec) }}{\text { Runtime(HM Dec) }}\right)$ of our proposal versus the HM software in Table III. The left part of the table also shows the memory and complexity overhead of detecting and matching ORB and CNN features. Interestingly, the average decoding time of HMS is lower than HM. We attribute this reduction to the corresponding reduction of the average bitstream size allowed by our proposal (Table II). It has been independently reported [56] that BD-rate savingsor lead to reduced average decoding times as they allow for, on average, lower usage of the entropy decoding module (CABAC decoder), as well as the use of higher QP values. These effects lead to less computations per macroblock [56]. Since the reference HEVC implementation [27] does not allow for multithreaded execution, the reported times and RER/RDR correspond to single-threaded execution on an Intel Xeon Gold5120 CPU. As such, these comparisons should be taken as indicative of the overhead of our approach in a non-optimized software implementation and may translate to different relative runtimes under hardware-optimized encoding and decoding. In Fig. 6, we show that RER increases linearly with the number of stitching frames $N$. Importantly, as shown in Table III, the complexity associated with sequence-level reference selection exists only during encoding; this complexity overhead is not relevant to the decoder. 
TABLE II: Bjontegaard delta rate (\%), delta PSNR, and delta VMAF on synthesized standard definition videos. Each synthesized video of SV1-SV6 and BSH1-BSH3 is made by interleaving $R$ randomly-selected segments from $K$ distinct source videos.

\begin{tabular}{|c|c|c|c|c|c|c|c|c|c|}
\hline \multirow{2}{*}{$\begin{array}{c}\text { Sequence } \\
(K, R)\end{array}$} & \multirow[b]{2}{*}{ Anchor } & \multicolumn{4}{|c|}{ HMS } & \multicolumn{4}{|c|}{ HMS-PBBB } \\
\hline & & BD-Rate $_{P}$ & BD-PSNR & BD-Rate $_{\mathrm{v}}$ & BD-VMAF & BD-Rate $_{\mathrm{P}}$ & BD-PSNR & BD-Rate $_{v}$ & BD-VMAF \\
\hline \multirow{3}{*}{$\begin{array}{c}\text { SV1 } \\
(2,10)\end{array}$} & HM & $-18.14 \%$ & $0.86 \mathrm{~dB}$ & $-20.08 \%$ & 1.17 & $-30.86 \%$ & $1.60 \mathrm{~dB}$ & $-26.98 \%$ & 1.77 \\
\hline & VP9 & $-17.01 \%$ & $0.77 \mathrm{~dB}$ & $-25.95 \%$ & 1.61 & $-30.23 \%$ & $1.50 \mathrm{~dB}$ & $-37.05 \%$ & 2.13 \\
\hline & HM-PBBB & $-29.29 \%$ & $1.39 \mathrm{~dB}$ & $-6.73 \%$ & 0.95 & $-40.38 \%$ & $2.11 \mathrm{~dB}$ & $-31.46 \%$ & 1.69 \\
\hline \multirow{3}{*}{$\begin{array}{c}\text { SV2 } \\
(2,20)\end{array}$} & HM & $-32.12 \%$ & $1.76 \mathrm{~dB}$ & $-28.71 \%$ & 3.69 & $-36.39 \%$ & $2.11 \mathrm{~dB}$ & $-33.58 \%$ & 4.35 \\
\hline & VP9 & $-28.96 \%$ & $1.60 \mathrm{~dB}$ & $-27.17 \%$ & 2.82 & $-33.49 \%$ & $1.97 \mathrm{~dB}$ & $-31.61 \%$ & 3.41 \\
\hline & HM-РВBВ & $-27.89 \%$ & $1.48 \mathrm{~dB}$ & $-12.87 \%$ & 1.35 & $-32.51 \%$ & $1.84 \mathrm{~dB}$ & $-19.19 \%$ & 2.01 \\
\hline \multirow{3}{*}{$\begin{array}{c}\text { SV3 } \\
(3,12)\end{array}$} & HM & $-29.86 \%$ & $1.65 \mathrm{~dB}$ & $-11.06 \%$ & 3.51 & $-36.55 \%$ & $2.15 \mathrm{~dB}$ & $-4.08 \%$ & 4.04 \\
\hline & VP9 & $-30.79 \%$ & $1.66 \mathrm{~dB}$ & $-17.99 \%$ & 3.76 & $-37.43 \%$ & $2.18 \mathrm{~dB}$ & $-11.47 \%$ & 4.22 \\
\hline & HM-PBBB & $-41.31 \%$ & $2.30 \mathrm{~dB}$ & $-9.09 \%$ & 2.32 & $-46.76 \%$ & $2.80 \mathrm{~dB}$ & $-4.52 \%$ & 2.94 \\
\hline \multirow{3}{*}{$\begin{array}{c}\text { SV4 } \\
(3,12)\end{array}$} & HM & $-18.37 \%$ & $0.92 \mathrm{~dB}$ & $-16.60 \%$ & 2.62 & $-25.85 \%$ & $1.37 \mathrm{~dB}$ & $-18.78 \%$ & 3.26 \\
\hline & VP9 & $-21.70 \%$ & $1.07 \mathrm{~dB}$ & $-23.41 \%$ & 2.96 & $-28.94 \%$ & $1.53 \mathrm{~dB}$ & $-25.79 \%$ & 3.54 \\
\hline & HM-РBBB & $-29.27 \%$ & $1.45 \mathrm{~dB}$ & $-13.19 \%$ & 1.18 & $-35.86 \%$ & $1.90 \mathrm{~dB}$ & $-15.46 \%$ & 1.89 \\
\hline \multirow{3}{*}{$\begin{array}{l}\text { SV5 } \\
(3,20)\end{array}$} & HM & $-20.44 \%$ & $1.05 \mathrm{~dB}$ & $-12.46 \%$ & 2.72 & $-27.73 \%$ & $1.51 \mathrm{~dB}$ & $-12.54 \%$ & 3.34 \\
\hline & VP9 & $-22.08 \%$ & $1.10 \mathrm{~dB}$ & $-19.04 \%$ & 3.13 & $-29.30 \%$ & $1.58 \mathrm{~dB}$ & $-19.69 \%$ & 3.70 \\
\hline & HМ-РВBВ & $-23.25 \%$ & $1.15 \mathrm{~dB}$ & $-10.46 \%$ & 1.13 & $-30.27 \%$ & $1.60 \mathrm{~dB}$ & $-8.68 \%$ & 1.80 \\
\hline \multirow{3}{*}{$\begin{array}{l}\text { SV6 } \\
(3,40)\end{array}$} & HM & $-32.55 \%$ & $1.70 \mathrm{~dB}$ & $-28.12 \%$ & 3.63 & $-40.15 \%$ & $2.23 \mathrm{~dB}$ & $-35.51 \%$ & 4.49 \\
\hline & VP9 & $-34.09 \%$ & $1.81 \mathrm{~dB}$ & $-32.43 \%$ & 3.99 & $-41.42 \%$ & $2.35 \mathrm{~dB}$ & $-38.51 \%$ & 4.71 \\
\hline & HM-РВBВ & $-24.63 \%$ & $1.22 \mathrm{~dB}$ & $-13.31 \%$ & 1.19 & $-33.15 \%$ & $1.76 \mathrm{~dB}$ & $-20.51 \%$ & 2.07 \\
\hline \multirow{3}{*}{$\begin{array}{l}\text { BSH1 } \\
(3,10)\end{array}$} & $\mathrm{HM}$ & $-20.73 \%$ & $0.73 \mathrm{~dB}$ & $-26.89 \%$ & 5.17 & $-27.95 \%$ & $1.02 \mathrm{~dB}$ & $-48.56 \%$ & 9.44 \\
\hline & VP9 & $-40.05 \%$ & $1.80 \mathrm{~dB}$ & $-49.84 \%$ & 12.08 & $-47.15 \%$ & $1.98 \mathrm{~dB}$ & $-64.48 \%$ & 14.58 \\
\hline & НM-РВBВ & $-16.11 \%$ & $0.56 \mathrm{~dB}$ & $-1.07 \%$ & 0.11 & $-22.93 \%$ & $0.83 \mathrm{~dB}$ & $-29.26 \%$ & 4.68 \\
\hline \multirow{3}{*}{$\begin{array}{l}\text { BSH2 } \\
(3,15)\end{array}$} & HM & $-30.32 \%$ & $1.06 \mathrm{~dB}$ & $-31.14 \%$ & 6.50 & $-38.29 \%$ & $1.33 \mathrm{~dB}$ & $-50.52 \%$ & 11.46 \\
\hline & VP9 & $-47.03 \%$ & $2.05 \mathrm{~dB}$ & $-53.06 \%$ & 14.84 & $-52.62 \%$ & $2.12 \mathrm{~dB}$ & $-67.56 \%$ & 17.83 \\
\hline & HM-PBBB & $-20.39 \%$ & $0.66 \mathrm{~dB}$ & $-4.13 \%$ & 0.58 & $-28.80 \%$ & $0.87 \mathrm{~dB}$ & $-37.82 \%$ & 5.36 \\
\hline \multirow{3}{*}{$\begin{array}{l}\text { BSH3 } \\
(3,20)\end{array}$} & HM & $-34.05 \%$ & $1.19 \mathrm{~dB}$ & $-37.47 \%$ & 9.54 & $-40.49 \%$ & $1.34 \mathrm{~dB}$ & $-52.49 \%$ & 12.75 \\
\hline & VP9 & $-48.07 \%$ & $1.87 \mathrm{~dB}$ & $-56.14 \%$ & 15.26 & $-54.82 \%$ & $2.15 \mathrm{~dB}$ & $-66.02 \%$ & 18.85 \\
\hline & HM-PBBB & $-23.32 \%$ & $0.67 \mathrm{~dB}$ & $-7.18 \%$ & 1.20 & $-35.92 \%$ & $1.14 \mathrm{~dB}$ & $-41.57 \%$ & 7.44 \\
\hline
\end{tabular}

\section{B. Evaluation On Natural Videos and Performance of ORB Versus $C N N$}

Beyond synthesized videos, we test our approach on natural videos from FILMShort [50] and high-definition videos from the UVG [49] public repository under the same encoding configuration of Section V.A. Specifically, we compare the performance of all methods on the following publicly available videos $^{4}$ [50]: "Breathe", "City Lights", "Jet", "Naughty List", "Big Bunny", and "Brink". For the natural sequences of FILMShort [50], we select our test videos to cover a wide range of semantic repetition, and in Table I we detail the degree of semantic repetition in each video. By doing so, we measure the benefit of extending the referencing period of inter-frame prediction when different degrees of repetition exist across scenes.

We report the corresponding Bjontegaard deltas in Table IV, where we also compare the performance of the lowcomplexity ORB-based variant of our method (denoted as HMS) to the more complex CNN-based variant HMS-CNN proposed in Section IV. From Table IV, we first observe that HMS performs comparably to HMS-CNN, with two

\footnotetext{
${ }^{4} \mathrm{We}$ provide links to retrieve all videos in https://github.com/ hevc-extended-term-frame-referencing
}

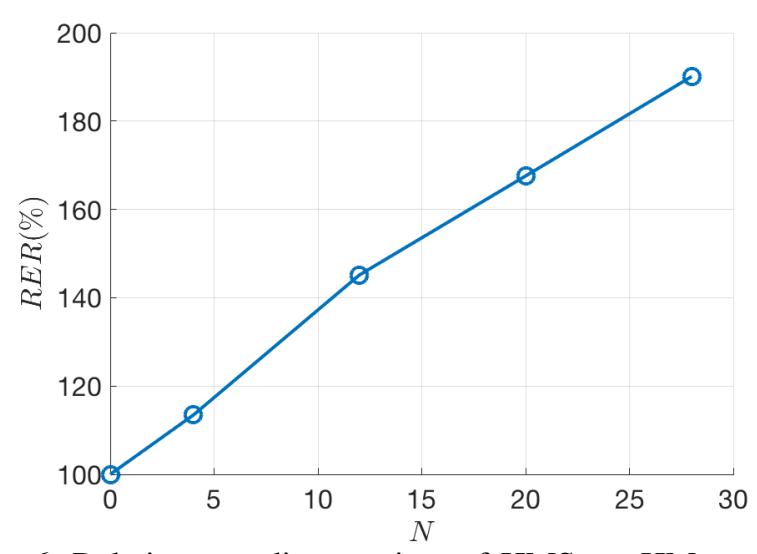

Fig. 6: Relative encoding runtime of HMS vs. HM encoding when varying the number of sequence-level references $N$. Note that the increase in RER is linear to $N$.

exceptions being on the sequences "Naughty List" and "Jet", where HMS-CNN performs with a higher margin relative to other sequences. For example, in terms of the rate-PSNR performance of "Brink", HMS provides BD-Rate savings of up to $37.41 \%$ and $25.62 \%$ over HM and VP9, respectively, while HMS-CNN provides savings of $38.05 \%$ and $30.34 \%$ on 
TABLE III: Average runtime and memory requirements for sequence-level reference selection and HMS encoding and decoding corresponding to the experiments of Table II. Reported are the averages of: runtime, relative encoding runtime (RER), relative decoding runtime (RDR), and memory usage for the proposed HMS encoding and decoding. 'Runtime-ORB' and 'RuntimeCNN' denote the average runtime for rate prediction via ORB and the proposed CNN approaches. 'Memory' for HMS encoding reports the average memory per frame inclusive of all encoding components, and all references used.

\begin{tabular}{|c|c|c|c|c|c|c|c|c|c|c|c|}
\hline \multirow[b]{2}{*}{ Sequence } & \multicolumn{4}{|c|}{ Sequence-level Reference Selection } & \multicolumn{3}{|c|}{ HMS Encoding } & \multicolumn{4}{|c|}{ HMS Decoding } \\
\hline & $(M, N)$ & $\begin{array}{l}\text { Runtime-ORB } \\
\text { (sec/frame) }\end{array}$ & $\begin{array}{l}\text { Runtime-CNN } \\
\text { (sec/frame) }\end{array}$ & $\begin{array}{l}\text { Memory } \\
\text { (GB) }\end{array}$ & $\begin{array}{l}\text { Runtime } \\
\text { (sec/frame) }\end{array}$ & $\begin{array}{l}\text { RER } \\
(\%)\end{array}$ & $\begin{array}{c}\text { Memory } \\
\text { (MB/frame) }\end{array}$ & $\begin{array}{c}\text { Runtime } \\
\text { (msec/frame) }\end{array}$ & $\begin{array}{l}\text { RDR } \\
(\%)\end{array}$ & (MB) & $\begin{array}{l}\text { Memory } \\
\text { (MB/frame) }\end{array}$ \\
\hline SV1 & $(400,16)$ & 2.035 & 10.298 & 5.758 & 6.888 & $131.1 \%$ & 3.320 & 5.592 & 81.281 & 96 & 0.2400 \\
\hline SV2 & $(800,20)$ & 3.302 & 12.873 & 6.062 & 8.313 & $160.0 \%$ & 3.456 & 6.238 & 81.535 & 154 & 0.1925 \\
\hline SV3 & $(800,28)$ & 3.850 & 18.023 & 5.783 & 9.086 & $182.1 \%$ & 3.712 & 6.426 & 82.955 & 192 & 0.2400 \\
\hline SV4 & $(800,25)$ & 3.570 & 16.092 & 5.986 & 8.620 & $165.3 \%$ & 3.584 & 6.313 & 83.122 & 180 & 0.2250 \\
\hline SV5 & $(1200,44)$ & 7.381 & 28.322 & 7.520 & 11.547 & $208.3 \%$ & 4.352 & 6.670 & 84.120 & 299 & 0.2492 \\
\hline SV6 & $(2000,52)$ & 8.540 & 33.471 & 10.557 & 13.574 & $251.7 \%$ & 7.786 & 6.524 & 83.384 & 394 & 0.1970 \\
\hline BSH1 & $(1000,23)$ & 3.370 & 14.804 & 6.527 & 25.067 & $170.2 \%$ & 3.552 & 42.604 & 81.512 & 994 & 0.9940 \\
\hline BSH2 & $(1600,36)$ & 7.581 & 23.173 & 9.032 & 29.006 & $194.7 \%$ & 4.223 & 42.830 & 84.347 & 1537 & 0.9606 \\
\hline BSH3 & $(2000,47)$ & 8.040 & 30.253 & 10.147 & 33.241 & $226.3 \%$ & 7.352 & 44.842 & 83.577 & 2046 & 1.0230 \\
\hline
\end{tabular}

TABLE IV: Bjontegaard delta rate (\%), delta PSNR, and delta VMAF for HMS and HMS-CNN on natural Standard Definition videos from the FILMshort [50] and UVG [49] repositories. Note that GOP-LTP [33] results are excluded for \{Bosphorus, ShakeNDry,HoneyBee\} due to their lacking of multiple-scenes which is a prerequisite for GOP-LTP [33].

\begin{tabular}{|c|c|c|c|c|c|c|c|c|c|}
\hline \multirow{2}{*}{$\begin{array}{c}\text { Natural } \\
\text { Sequence }\end{array}$} & \multirow[b]{2}{*}{ Anchor } & \multicolumn{4}{|c|}{ HMS } & \multicolumn{4}{|c|}{ HMS-CNN } \\
\hline & & BD-Rate $_{\mathrm{P}}$ & BD-PSNR & BD-Rate $_{\mathrm{v}}$ & BD-VMAF & BD-Rate $_{P}$ & BD-PSNR & BD-Rate $_{v}$ & BD-VMAF \\
\hline \multirow{3}{*}{ Breathe } & $\mathrm{HM}$ & $-24.81 \%$ & $1.17 \mathrm{~dB}$ & $-25.22 \%$ & 2.93 & $-28.62 \%$ & $1.32 \mathrm{~dB}$ & $-25.70 \%$ & 3.35 \\
\hline & VP9 & $-16.18 \%$ & $0.73 \mathrm{~dB}$ & $-41.14 \%$ & 6.38 & $-19.52 \%$ & $0.85 \mathrm{~dB}$ & $-41.75 \%$ & 6.86 \\
\hline & GOP-LTP [33] & $-7.11 \%$ & $0.30 \mathrm{~dB}$ & $-21.12 \%$ & 3.78 & $-12.21 \%$ & $0.52 \mathrm{~dB}$ & $-21.81 \%$ & 4.34 \\
\hline \multirow{3}{*}{ City Lights } & $\mathrm{HM}$ & $-34.44 \%$ & $1.94 \mathrm{~dB}$ & $-29.33 \%$ & 3.21 & $-34.71 \%$ & $1.92 \mathrm{~dB}$ & $-31.69 \%$ & 3.65 \\
\hline & VP9 & $-37.37 \%$ & $2.21 \mathrm{~dB}$ & $-53.84 \%$ & 9.24 & $-39.07 \%$ & $2.44 \mathrm{~dB}$ & $-54.61 \%$ & 9.63 \\
\hline & GOP-LTP [33] & $-3.37 \%$ & $0.18 \mathrm{~dB}$ & $-9.46 \%$ & 1.83 & $-7.69 \%$ & $0.45 \mathrm{~dB}$ & $-16.61 \%$ & 2.70 \\
\hline \multirow{3}{*}{ Brink } & $\mathrm{HM}$ & $-37.41 \%$ & $2.06 \mathrm{~dB}$ & $-24.65 \%$ & 3.25 & $-38.05 \%$ & $2.26 \mathrm{~dB}$ & $-3.64 \%$ & 3.32 \\
\hline & VP9 & $-25.62 \%$ & $1.34 \mathrm{~dB}$ & $-39.97 \%$ & 6.44 & $-30.34 \%$ & $1.70 \mathrm{~dB}$ & $-32.04 \%$ & 6.72 \\
\hline & GOP-LTP [33] & $-14.34 \%$ & $0.78 \mathrm{~dB}$ & $-22.62 \%$ & 3.94 & $-18.59 \%$ & $1.05 \mathrm{~dB}$ & $-8.03 \%$ & 5.04 \\
\hline \multirow{3}{*}{ Naught List } & $\mathrm{HM}$ & $-1.29 \%$ & $0.04 \mathrm{~dB}$ & $-17.92 \%$ & 2.39 & $-7.12 \%$ & $0.32 \mathrm{~dB}$ & $-30.69 \%$ & 4.53 \\
\hline & VP9 & $-23.48 \%$ & $1.27 \mathrm{~dB}$ & $-43.63 \%$ & 8.52 & $-28.21 \%$ & $1.62 \mathrm{~dB}$ & $-52.27 \%$ & 11.57 \\
\hline & GOP-LTP [33] & $-15.24 \%$ & $0.68 \mathrm{~dB}$ & $-19.99 \%$ & 2.44 & $-4.14 \%$ & $0.19 \mathrm{~dB}$ & $-18.70 \%$ & 2.40 \\
\hline \multirow{3}{*}{ Jet } & $\mathrm{HM}$ & $-3.98 \%$ & $0.14 \mathrm{~dB}$ & $-25.27 \%$ & 2.23 & $-14.24 \%$ & $0.62 \mathrm{~dB}$ & $-25.55 \%$ & 1.72 \\
\hline & VP9 & $-30.58 \%$ & $1.78 \mathrm{~dB}$ & $-50.53 \%$ & 9.65 & $-39.84 \%$ & $2.38 \mathrm{~dB}$ & $-49.02 \%$ & 9.50 \\
\hline & GOP-LTP [33] & $-10.27 \%$ & $0.47 \mathrm{~dB}$ & $-27.75 \%$ & 3.12 & $-23.81 \%$ & $1.22 \mathrm{~dB}$ & $-23.79 \%$ & 3.06 \\
\hline \multirow{3}{*}{ Big Bunny } & HM & $-13.42 \%$ & $0.83 \mathrm{~dB}$ & $-20.38 \%$ & 3.23 & $-16.34 \%$ & $0.94 \mathrm{~dB}$ & $-21.72 \%$ & 3.60 \\
\hline & VP9 & $-31.14 \%$ & $1.93 \mathrm{~dB}$ & $-41.11 \%$ & 7.08 & $-31.47 \%$ & $1.99 \mathrm{~dB}$ & $-41.46 \%$ & 8.15 \\
\hline & GOP-LTP [33] & $-3.15 \%$ & $0.18 \mathrm{~dB}$ & $-6.92 \%$ & 1.80 & $-3.65 \%$ & $0.20 \mathrm{~dB}$ & $-6.44 \%$ & 1.52 \\
\hline \multirow[t]{2}{*}{ Bosphorus } & $\mathrm{HM}$ & $-4.07 \%$ & $0.11 \mathrm{~dB}$ & $-0.73 \%$ & 0.09 & $-5.60 \%$ & $0.16 \mathrm{~dB}$ & $-1.26 \%$ & 0.17 \\
\hline & VP9 & $-19.02 \%$ & $0.66 \mathrm{~dB}$ & $-36.17 \%$ & 7.83 & $-20.21 \%$ & $0.71 \mathrm{~dB}$ & $-36.56 \%$ & 7.91 \\
\hline \multirow[t]{2}{*}{ ShakeNDry } & HM & $-6.14 \%$ & $0.12 \mathrm{~dB}$ & $-8.45 \%$ & 1.24 & $-9.36 \%$ & $0.19 \mathrm{~dB}$ & $-7.61 \%$ & 1.10 \\
\hline & VP9 & $-16.13 \%$ & $0.39 \mathrm{~dB}$ & $-33.85 \%$ & 6.62 & $-18.75 \%$ & $0.46 \mathrm{~dB}$ & $-33.24 \%$ & 6.33 \\
\hline \multirow[t]{2}{*}{ HoneyBee } & $\mathrm{HM}$ & $-19.46 \%$ & $0.39 \mathrm{~dB}$ & $-9.25 \%$ & 0.43 & $-25.73 \%$ & $0.52 \mathrm{~dB}$ & $-9.57 \%$ & 0.49 \\
\hline & VP9 & $-30.10 \%$ & $0.07 \mathrm{~dB}$ & $-53.65 \%$ & 2.53 & $-31.56 \%$ & $0.09 \mathrm{~dB}$ & $-54.36 \%$ & 2.57 \\
\hline
\end{tabular}

the same comparison. Relative improvement is also seen in the corresponding gains in PSNR, and we observe similar results with respect to VMAF. Moreover, comparisons against VP9 reliably show that HMS and HMS-CNN both perform better than VP9 on all test sequences, and by up to $37.37 \%$ and $30.58 \%$ BD-Rate savings for HMS on the sequences "City Lights" and "Jet", respectively. The latter gives a strong indication that sequence-level references provide useful semantics for inter-frame prediction that augment the benefit of summarised frames enabled in VP9. Crucially, the small relative gains in performance between HMS and HMS-CNN indicate that the low-complexity variant HMS (based on ORB) suffices for most sequences, while HMS-CNN can be used whenever its complexity requirements can be met to produce slightly better results across all reported metrics.

Compared to GOP-LTP [33], which is the work closest to ours, we observe that both HMS and HMS-CNN outperform the HM adaptation of GOP-LTP [33] by significant margins of up to $23.81 \%$ BD-Rate savings for the "Jet" sequence. This gain in performance is attributed two important distinctions of our work to GOP-LTP [33]: (i) GOP-LTP is limited to selecting scene-cut frames as LTRs, while ours considers all frames as potential LTRs, and (ii) distances in GOP-LTP are only measured among scene-cut frames, while our method 
TABLE V: Bjontegaard delta rate (\%), delta PSNR, and delta VMAF for VVCS as tested on natural video sequences from the Ultra Video Group repository [49].

\begin{tabular}{|c|c|c|c|c|c|c|c|c|c|}
\hline \multirow[b]{2}{*}{ Sequence } & \multirow[b]{2}{*}{ Anchor } & \multicolumn{4}{|c|}{ VVCS } & \multicolumn{4}{|c|}{ VVCS-PBBB } \\
\hline & & BD-Rate $_{\mathrm{P}}$ & BD-PSNR & BD-Rate $_{\mathrm{v}}$ & BD-VMAF & BD-Rate $_{\mathrm{P}}$ & BD-PSNR & BD-Rate $_{v}$ & BD-VMAF \\
\hline \multirow[t]{2}{*}{ Bosphorus } & VVC & $-1.54 \%$ & $0.037 \mathrm{~dB}$ & $-1.15 \%$ & 0.129 & $-4.37 \%$ & $0.112 \mathrm{~dB}$ & $-2.44 \%$ & 0.352 \\
\hline & VVC-PBBB & $1.39 \%$ & $-0.048 \mathrm{~dB}$ & $0.13 \%$ & -0.071 & $-1.52 \%$ & $0.026 \mathrm{~dB}$ & $-1.16 \%$ & 0.152 \\
\hline \multirow[t]{2}{*}{ ShakeNDry } & VVC & $-16.21 \%$ & $0.324 \mathrm{~dB}$ & $-3.57 \%$ & 0.485 & $-19.33 \%$ & $0.377 \mathrm{~dB}$ & $-7.24 \%$ & 0.963 \\
\hline & VVC-PBBB & $-16.01 \%$ & $0.294 \mathrm{~dB}$ & $-3.14 \%$ & 0.385 & $-19.10 \%$ & $0.353 \mathrm{~dB}$ & $-6.59 \%$ & 0.808 \\
\hline \multirow[t]{2}{*}{ HoneyBee } & VVC & $-2.84 \%$ & $0.023 \mathrm{~dB}$ & $-1.63 \%$ & 0.053 & $-5.78 \%$ & $0.046 \mathrm{~dB}$ & $-2.44 \%$ & 0.042 \\
\hline & VVC-PBBB & $0.67 \%$ & $-0.002 \mathrm{~dB}$ & $0.49 \%$ & -0.003 & $-2.39 \%$ & $0.020 \mathrm{~dB}$ & $-0.21 \%$ & 0.013 \\
\hline
\end{tabular}

TABLE VI: Bjontegaard delta rate (\%), delta PSNR, and delta VMAF for VVCS as tested on synthesized video sequences from the Ultra Video Group repository [49].

\begin{tabular}{|c|c|c|c|c|c|c|c|c|c|}
\hline \multirow[b]{2}{*}{ Sequence } & \multirow[b]{2}{*}{ Anchor } & \multicolumn{4}{|c|}{ VVCS } & \multicolumn{4}{|c|}{ VVCS-PBBB } \\
\hline & & BD-Rate $_{P}$ & BD-PSNR & BD-Rate $_{\mathrm{v}}$ & BD-VMAF & BD-Rate $_{P}$ & BD-PSNR & BD-Rate $_{\mathrm{v}}$ & BD-VMAF \\
\hline BSH1 & VVC & $-17.63 \%$ & $0.623 \mathrm{~dB}$ & $-20.82 \%$ & 4.325 & $-20.18 \%$ & $0.709 \mathrm{~dB}$ & $-24.21 \%$ & 4.863 \\
\hline$(3,10)$ & VVC-PBBB & $-17.12 \%$ & $0.525 \mathrm{~dB}$ & $-20.11 \%$ & 2.663 & $-16.48 \%$ & $0.491 \mathrm{~dB}$ & $-21.00 \%$ & 2.676 \\
\hline BSH2 & VVC & $-31.54 \%$ & $1.000 \mathrm{~dB}$ & $-28.172 \%$ & 4.022 & $-32.53 \%$ & $1.076 \mathrm{~dB}$ & $-28.99 \%$ & 4.165 \\
\hline$(3,15)$ & VVC-PBBB & $-31.51 \%$ & $0.996 \mathrm{~dB}$ & $-27.047 \%$ & 3.865 & $-32.50 \%$ & $1.072 \mathrm{~dB}$ & $-27.88 \%$ & 4.009 \\
\hline BSH3 & VVC & $-28.75 \%$ & $0.885 \mathrm{~dB}$ & $-30.35 \%$ & 5.362 & $-30.52 \%$ & $0.947 \mathrm{~dB}$ & $-34.45 \%$ & 6.160 \\
\hline$(3,20)$ & VVC-PBBB & $-27.87 \%$ & $0.884 \mathrm{~dB}$ & $-26.49 \%$ & 4.795 & $-29.62 \%$ & $0.949 \mathrm{~dB}$ & $-30.44 \%$ & 5.594 \\
\hline
\end{tabular}

considers the distance between LTR candidates and all frames in the video. Notice that GOP-LTP requires the availability of multiple scene-cut frames, and therefore cannot be validated on the short sequences $\{$ Bosphorus, ShakeNDry, HoneyBee\} comprising singular scenes.

Indicative quality-bitrate curves for the non-hierarchical IPPP structure are shown in Fig. 7. Notably, we see that the PSNR values of HMS are significantly higher than those of HM and VP9 across all average bitrate values, and HMS can outperform HM by up to $2.8 \mathrm{~dB}$ and $2.1 \mathrm{~dB}$ when considering "City Lights" and "Brink", respectively. With regards to VMAF, we see that, unlike PSNR, HMS provides diminishing improvements over HM and VP9 for higher bitrates. This is due to the saturation property intrinsic to the high regime of VMAF, which reflects the fact that the increase in perceptual quality of high-bitrate video is not linearly correlated with the reduction in the power of the reconstruction error [54].

\section{Evaluation on Versatile Video Coding}

In order to validate the gains provided by our proposal with respect to next-generation video coding, we report a first comparison against the recently-completed VVC standard of MPEG/ITU-T. In order to test the extent of savings achievable by including sequence-level references in VVC [34], which we hereon denote as VVC-Stitching (VVCS), in Table V and Table VI we report BD-rates achieved against the VVC Test Model (VTM) 7.1. We set the VTM coding profile to the default setting "auto", and present our tests for three HD videos from the UVG repository [49], namely: "Bosphorus",
"ShakeNDry", and "HoneyBee". 5 Given that the short playtime of standard videos in the UVG repository impedes the full extent of rate reduction by sequence-level referencing, we additionally report gains when repetition is artificially introduced by synthesizing all video sequences with prespecified parameters $K$ and $R$. Table $\mathrm{V}$ shows that sequencelevel references provide modest rate savings for the short and non-repetitive video sequences "Bosphorus", "ShakeNDry", and "HoneyBee". However, the reported gains are comparable or superior to those of tools that have been considered for inclusion in the VVC standard [34]. For example, Pfaff et al. [57] present an analysis showing that 8 out of the 14 new coding tools (Table III of their paper [57]) achieve BD-rates of $-0.7 \%$ to $-2.8 \%$, but they cumulatively contribute to BDrate of $-21 \%$ over the HM reference software. Such scaleup of performance is observed in our proposal when scene repetition occurs more frequently in the input video: Table VI shows that sequence-level references provide for up to $-33 \%$ in BD-rate for PSNR or VMAF in comparison to VVC. Further evaluation will be possible when the VTM reference software matures further to allow for faster encoding, which will allow for multi-bitrate encodings of longer video sequences with our proposal.

\section{CONCLUSION}

We propose sequence-level references for inter-frame video coding. In our approach, reference frames are selected by first mapping them in a compact descriptor space, thereby making

\footnotetext{
${ }^{5}$ At the time of this writing, VTM 7.1 is extremely slow, i.e., it takes several minutes to encode a single frame. This constraint, in conjunction with the fact that the VTM is still undergoing revisions to improve the performance of its various encoding tools, limits the extent of our test.
} 


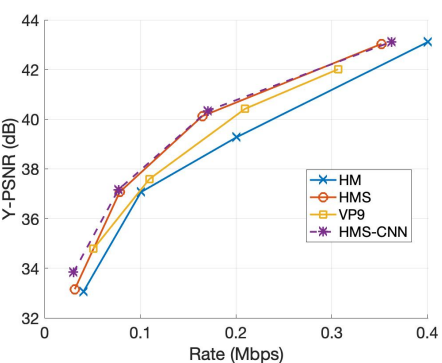

(a) Breathe $(\mathrm{N}=56)$

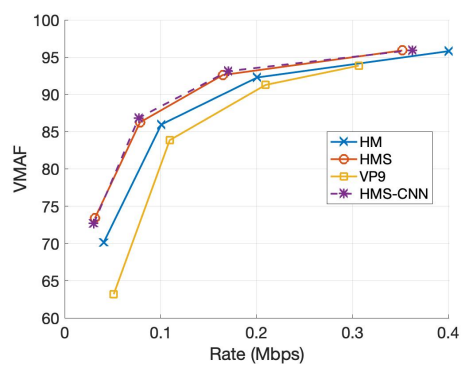

(e) Breathe $(\mathrm{N}=56)$

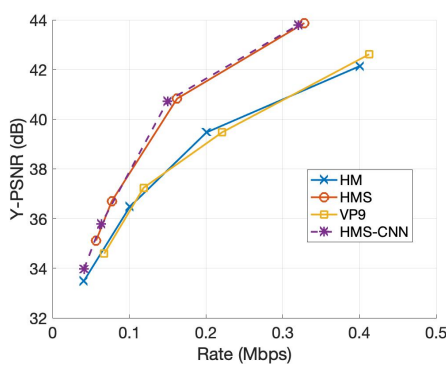

(b) City Lights $(\mathrm{N}=56)$

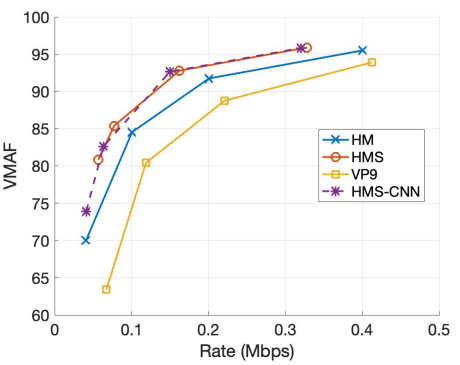

(f) City Lights $(\mathrm{N}=56)$

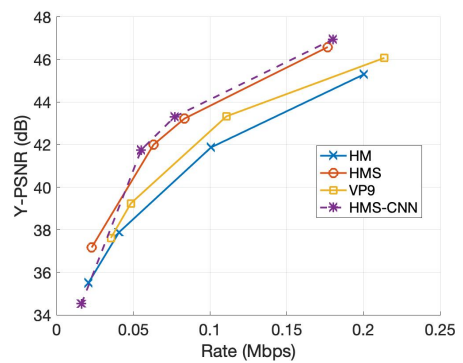

(c) Brink $(\mathrm{N}=56)$

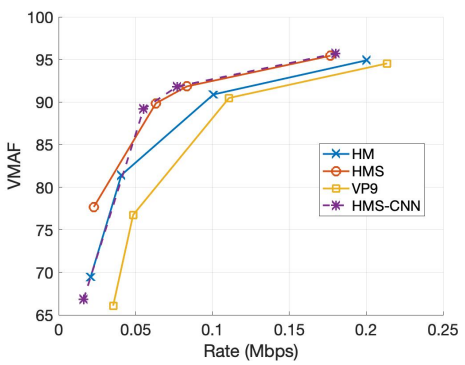

(g) Brink $(\mathrm{N}=56)$

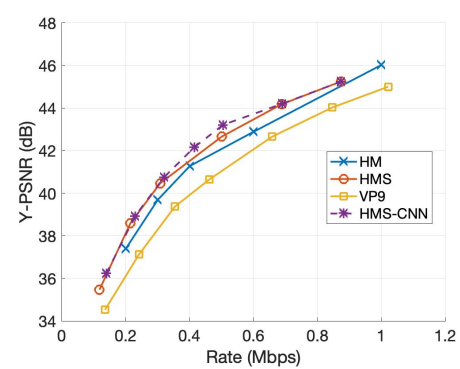

(d) Big Buck Bunny (N=34)

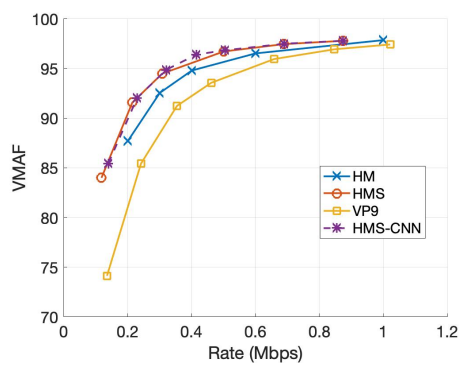

(h) Big Buck Bunny (N=34)

Fig. 7: Rate-PSNR and Rate-VMAF when content from FILMshort [50] is encoded using different bitrate targets. When sequence-level references are used, the number of active references used is set to $N+4$, where 4 is the number of predictors required by the default GOP structure.

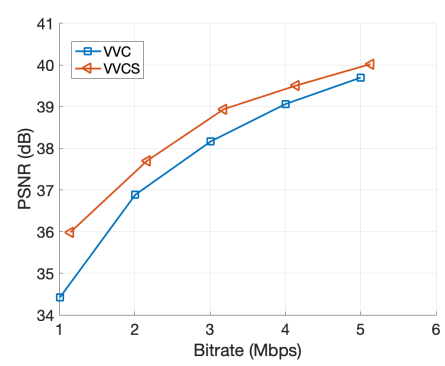

(a) $\operatorname{BSH}(3,10)(\mathrm{N}=23)$

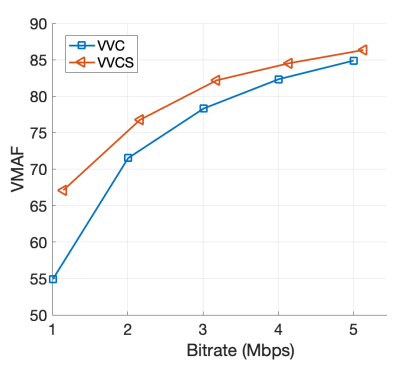

(b) $\operatorname{BSH}(3,10)(\mathrm{N}=23)$

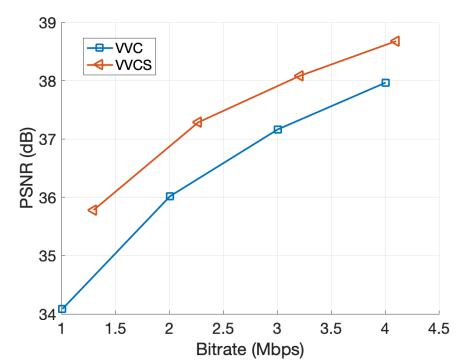

(c) $\operatorname{BSH}(3,20)(\mathrm{N}=47)$

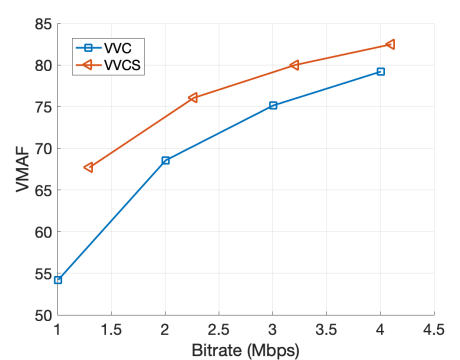

(d) $\operatorname{BSH}(3,20)(\mathrm{N}=47)$

Fig. 8: Rate-PSNR and Rate-VMAF when content from the Ultra Video Group [49] is encoded via VVC vs. VVCS, and using different bitrate targets. When sequence-level references are used, the number of active references is set to $N+4$, where 4 is the number of predictors required by the default GOP structure.

it possible to derive sequence-level references for long video sequences without increasing memory and computational requirements to infeasible levels. We demonstrate that our approach can be applied jointly with hierarchical and low-delay prediction structures in video coding without breaking decoder standard compliance. An instantiation of our method in the HEVC reference software is shown to outperform the reference HEVC and VP9 encoders for sequences with synthetic and naturally-occurring scene repetitions. Further validation on the VVC Test Model 7.1 shows that sequence-level references improves VVC's rate-distortion in a non-negligible manner, especially as scene repetitions become more frequent within the test content.

Finally, initial runtime benchmarks demonstrate that encoding runtime and memory requirements scale linearly with increas- ing numbers of sequence-level references. All content and tools used to reproduce our results are publicly available at https://github.com/hevc-extended-term-frame-referencing.

\section{REFERENCES}

[1] T. Wiegand, X. Zhang, and B. Girod, "Motion-compensating long-term memory prediction," in Proceedings of International Conference on Image Processing, vol. 2, Oct 1997, pp. 53-56 vol.2.

[2] D. Grois, T. Nguyen, and D. Marpe, "Performance comparison of av1, jem, vp9, and hevc encoders," in Applications of Digital Image Processing XL, vol. 10396. International Society for Optics and Photonics, 2018, p. 103960L.

[3] V. Chellappa, P. C. Cosman, and G. M. Voelker, "Dual frame motion compensation with uneven quality assignment," IEEE Transactions on Circuits and Systems for Video Technology, vol. 18, no. 2, pp. 249-256, Feb 2008. 
[4] M. Tiwari and P. C. Cosman, "Selection of long-term reference frames in dual-frame video coding using simulated annealing," IEEE Signal Processing Letters, vol. 15, pp. 249-252, 2008.

[5] D. Liu, D. Zhao, X. Ji, and W. Gao, "Dual frame motion compensation with optimal long-term reference frame selection and bit allocation," IEEE Transactions on Circuits and Systems for Video Technology, vol. 20, no. 3, pp. 325-339, March 2010.

[6] G. J. Sullivan, J.-R. Ohm, W.-J. Han, and T. Wiegand, "Overview of the high efficiency video coding (hevc) standard," IEEE Transactions on circuits and systems for video technology, vol. 22, no. 12, pp. 1649$1668,2012$.

[7] A. Muhammad and S. M. Daudpota, "Content based identification of talk show videos using audio visual features," in International Conference on Machine Learning and Data Mining in Pattern Recognition. Springer, 2016, pp. 267-283.

[8] B. Lehane, N. O'Connor, and N. Murphy, "Action sequence detection in motion pictures," in European Workshop on the Integration of Knowledge, Semantics and Digital Media Technology, 2004.

[9] J. E. Cutting, K. L. Brunick, J. E. DeLong, C. Iricinschi, and A. Candan, "Quicker, faster, darker: Changes in hollywood film over 75 years," $i$ Perception, vol. 2, no. 6, pp. 569-576, 2011.

[10] J. P. Hutson, T. J. Smith, J. P. Magliano, and L. C. Loschky, "What is the role of the film viewer? the effects of narrative comprehension and viewing task on gaze control in film," Cognitive Research: Principles and Implications, vol. 2, no. 1, p. 46, 2017.

[11] M. Muja and D. G. Lowe, "Fast matching of binary features," in 2012 Ninth Conference on Computer and Robot Vision, 2012, pp. 404-410.

[12] E. Rublee, V. Rabaud, K. Konolige, and G. Bradski, "Orb: An efficient alternative to sift or surf," in 2011 International Conference on Computer Vision, 2011, pp. 2564-2571.

[13] A. Chadha and Y. Andreopoulos, "Voronoi-based compact image descriptors: Efficient region-of-interest retrieval with vlad and deeplearning-based descriptors," IEEE Transactions on Multimedia, vol. 19, no. 7, pp. 1596-1608, 2017.

[14] Yu-Wen Huang, Bing-Yu Hsieh, Shao-Yi Chien, Shyh-Yih Ma, and Liang-Gee Chen, "Analysis and complexity reduction of multiple reference frames motion estimation in h.264/avc," IEEE Transactions on Circuits and Systems for Video Technology, vol. 16, no. 4, pp. 507-522, 2006.

[15] L. Shen, Z. Liu, Z. Zhang, and G. Wang, "An adaptive and fast multiframe selection algorithm for h.264 video coding," IEEE Signal Processing Letters, vol. 14, no. 11, pp. 836-839, Nov 2007.

[16] T. Kuo and H. Lu, "Efficient reference frame selector for h.264," IEEE Transactions on Circuits and Systems for Video Technology, vol. 18, no. 3, pp. 400-405, March 2008.

[17] Z. Liu, L. Li, Y. Song, S. Li, S. Goto, and T. Ikenaga, "Motion feature and hadamard coefficient-based fast multiple reference frame motion estimation for h.264," IEEE Transactions on Circuits and Systems for Video Technology, vol. 18, no. 5, pp. 620-632, May 2008.

[18] Y. Su and M.-T. Sun, "Fast multiple reference frame motion estimation for h.264/avc," IEEE Transactions on Circuits and Systems for Video Technology, vol. 16, no. 3, pp. 447-452, March 2006

[19] Y. Zhang, S. Kwong, G. Jiang, and H. Wang, "Efficient multi-reference frame selection algorithm for hierarchical $b$ pictures in multiview video coding," IEEE Transactions on Broadcasting, vol. 57, no. 1, pp. 15-23, 2010 .

[20] A. Chang, O. C. An, and Y. M. Yeung, "A novel approach to fast multiframe selection for h.264 video coding," in 2003 IEEE International Conference on Acoustics, Speech, and Signal Processing, 2003. Proceedings. (ICASSP '03)., vol. 3, 2003, pp. III-413.

[21] P. Wilkins, J. Bankoski, and Y. Xu, "System and method for video encoding using constructed reference frame," U.S. Patent 0061461 , Mar 2010.

[22] R. Ding, Q. Dai, W. Xu, D. Zhu, and H. Yin, "Background-frame based motion compensation for video compression," in 2004 IEEE International Conference on Multimedia and Expo (ICME) (IEEE Cat. No.04TH8763), vol. 2, June 2004, pp. 1487-1490 Vol.2

[23] K. Zhang and J. Kittler, "Using background memory for efficient video coding," in Proceedings 1998 International Conference on Image Processing. ICIP98 (Cat. No.98CB36269), Oct 1998, pp. 944-947 vol.3.

[24] M. Paul, W. Lin, C. Lau, and B. S. Lee, "A long-term reference frame for hierarchical b-picture-based video coding," IEEE Transactions on Circuits and Systems for Video Technology, vol. 24, no. 10, pp. 17291742, Oct 2014

[25] F. Chen, H. Li, L. Li, D. Liu, and F. Wu, "Block-composed background reference for high efficiency video coding," IEEE Transactions on
Circuits and Systems for Video Technology, vol. 27, no. 12, pp. 26392651, Dec 2017.

[26] C. Ma, D. Liu, X. Peng, F. Wu, H. Li, and T. Wang, "Reference clip for inter prediction in video coding," IEEE Transactions on Circuits and Systems for Video Technology, vol. 29, no. 1, pp. 130-143, Jan 2019.

[27] "HEVC reference software," https://hevc.hhi.fraunhofer.de/.

[28] D. Mukherjee, J. Bankoski, A. Grange, J. Han, J. Koleszar, P. Wilkins, $\mathrm{Y}$. $\mathrm{Xu}$, and R. Bultje, "The latest open-source video codec vp9-an overview and preliminary results," in 2013 Picture Coding Symposium (PCS). IEEE, 2013, pp. 390-393.

[29] D. Mukherjee, J. Han, J. Bankoski, R. Bultje, A. Grange, J. Koleszar, P. Wilkins, and Y. Xu, "A technical overview of vp9 the latest opensource video codec," in SMPTE 2013 Annual Technical Conference Exhibition, Oct 2013, pp. 1-17.

[30] W. Lin, Z. Liu, D. Mukherjee, J. Han, P. Wilkins, Y. Xu, and K. Rose, "Efficient av1 video coding using a multi-layer framework," in 2018 Data Compression Conference, March 2018, pp. 365-373.

[31] Z. Liu, D. Mukherjee, W. Lin, P. Wilkins, J. Han, and Y. Xu, "Adaptive multi-reference prediction using a symmetric framework," in Visual Information Processing and Communication, 2017.

[32] D. Chen, Z. Liu, F. Zhu, and E. J. Delp, "Multi-reference video coding using stillness detection," in Electronic Imaging, February 2018.

[33] X. Zuo and L. Yu, "Long-term prediction for hierarchical-b-picturebased coding of video with repeated shots," Frontiers Inf Technol Electronic Eng 19, pp. 459-470, 2018.

[34] "VVC/JVET reference software," https://jvet.hhi.fraunhofer.de/.

[35] A. Krizhevsky, I. Sutskever, and G. E. Hinton, "Imagenet classification with deep convolutional neural networks," in Advances in neural information processing systems, 2012, pp. 1097-1105.

[36] P. Isola, J.-Y. Zhu, T. Zhou, and A. A. Efros, "Image-to-image translation with conditional adversarial networks," in Proceedings of the IEEE conference on computer vision and pattern recognition, 2017, pp. 11251134.

[37] G. Koch, R. Zemel, and R. Salakhutdinov, "Siamese neural networks for one-shot image recognition," in ICML deep learning workshop, vol. 2. Lille, 2015.

[38] M. Mathias, R. Benenson, M. Pedersoli, and L. Van Gool, "Face detection without bells and whistles," in Computer Vision - ECCV 2014. Springer International Publishing, 2014, pp. 720-735.

[39] E. Karami, S. Prasad, and M. Shehata, "Image matching using sift, surf, brief and orb: performance comparison for distorted images," arXiv preprint arXiv:1710.02726, 2017.

[40] R. Arandjelovic, P. Gronat, A. Torii, T. Pajdla, and J. Sivic, "NetVLAD: CNN architecture for weakly supervised place recognition," in Proceedings of the IEEE Conference on Computer Vision and Pattern Recognition, 2016, pp. 5297-5307.

[41] Z. Xu, Y. Yang, and A. G. Hauptmann, "A discriminative cnn video representation for event detection," in Proceedings of the IEEE Conference on Computer Vision and Pattern Recognition, 2015, pp. 1798-1807.

[42] A. Abbas, N. Deligiannis, and Y. Andreopoulos, "Vectors of locally aggregated centers for compact video representation," in 2015 IEEE International Conference on Multimedia and Expo (ICME). IEEE, 2015, pp. $1-6$.

[43] W. Kay, J. Carreira, K. Simonyan, B. Zhang, C. Hillier, S. Vijayanarasimhan, F. Viola, T. Green, T. Back, P. Natsev et al., "The kinetics human action video dataset," arXiv preprint arXiv:1705.06950, 2017.

[44] L. Bottou, "Large-scale machine learning with stochastic gradient descent," in Proceedings of COMPSTAT'2010. Springer, 2010, pp. 177186

[45] A. Michele, V. Colin, and D. D. Santika, "MobileNet convolutional neural networks and support vector machines for palmprint recognition," Procedia Computer Science, vol. 157, pp. 110-117, 2019.

[46] J. Lin, Y. Rao, J. Lu, and J. Zhou, "Runtime neural pruning," in Advances in neural information processing systems, 2017, pp. 2181-2191.

[47] R. Setiono, "A penalty-function approach for pruning feedforward neural networks," Neural computation, vol. 9, no. 1, pp. 185-204, 1997.

[48] "Xiph video content repository," https://www.xiph.org/video/.

[49] A. Mercat, M. Viitanen, and J. Vanne, "Uvg dataset: 50/120fps 4k sequences for video codec analysis and development," Proceedings of the 11th ACM Multimedia Systems Conference, 2020.

[50] "Filmshort public short movie repository," https://www.filmsshort.com/.

[51] "Market share of top online video codecs and containers worldwide from 2016 to 2018," statista, May 2019.

[52] "PySceneDetect: An open source scene detection tool," https://github.com/Breakthrough/PySceneDetect/.

[53] "Ffmpeg transcoding tools," https://www.ffmpeg.org/. 
[54] Z. Li, A. Aaron, I. Katsavounidis, A. Moorthy, and M. Manohara, "Toward a practical perceptual video quality metric," in https://medium.com/netflix-techblog/toward-a-practical-perceptualvideo-quality-metric-653f208b9652, 20116.

[55] A. Rao, A. Legout, Y.-s. Lim, D. Towsley, C. Barakat, and W. Dabbous, "Network characteristics of video streaming traffic," in Proceedings of the Seventh COnference on emerging Networking EXperiments and Technologies, no. 25. ACM, December 2011, pp. 1-12.

[56] F. Bossen, B. Bross, K. Suhring, and D. Flynn, "Hevc complexity and implementation analysis," IEEE Transactions on Circuits and Systems for Video Technology, vol. 22, no. 12, pp. 1685-1696, 2012.

[57] J. Pfaff, H. Schwarz, D. Marpe, B. Bross, S. De-Luxán-Hernández, P. Helle, C. R. Helmrich, T. Hinz, W.-Q. Lim, J. Ma et al., "Video compression using generalized binary partitioning, trellis coded quantization, perceptually optimized encoding, and advanced prediction and transform coding," IEEE Transactions on Circuits and Systems for Video Technology, vol. 30, no. 5, pp. 1281-1295, 2019.

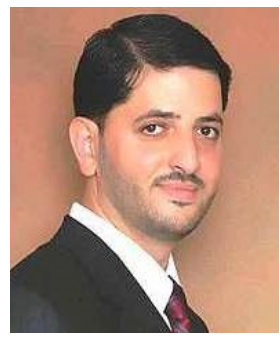

Mohammad Jubran is an Assistant Professor at the Department of Electrical and Computer Engineering at Birzeit University, Birzeit, Palestine. He has earned the B.S. degree in Electronic Engineering from Al-Quds University, Abu-Dies, Palestine, in 2000, and the M.S. and Ph.D. degrees in Electrical Engineering from the University at Buffalo, The State University of New York, Buffalo, NY, USA in 2005 and 2007, respectively. Dr. Jubran was a Visiting Postdoctoral Research Associate at University College London, London, UK (20172018) working on Video Processing, Communication and Classification using Machine Learning. Dr. Jubran is also an active member of IEEE and IEEE Communications Society. He has also served as the counselor of the IEEE student chapter at Birzeit University. Dr. Jubran research interests include video communication systems, wireless and mobile communications and machine learning. He published many articles in this area. $\mathrm{He}$ has also participated and led many international projects such as the e-Academy to support Smart Cities Operations in Palestine (eSCO) project funded by EC through the ERAMUS+ program. Dr. Jubran has received many regional and international awards such as the Distinguished Scholar Award - Arab Fund Program, Fulbright Scholarship, Zamalah Fellowship, and Academic Development Award from Birzeit University.

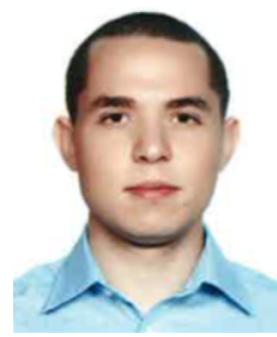

Alhabib Abbas obtained his BSc in Electrical and Electronic Engineering from the University of Bahrain, his MSc in Telecommunications from University College London (UCL), U.K. and the PhD in Electronic Engineering from UCL. He is currently a post-doctoral researcher in the Electronic Engineering Department of UCL. His research interests are in machine learning, image and video semantic analysis, and error-tolerant computing in multimedia systems.

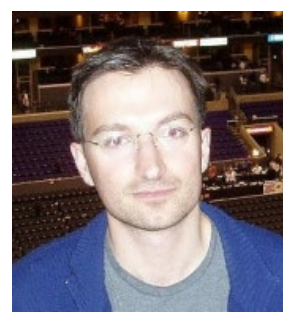

Yiannis Andreopoulos obtained the Electrical Engineering Diploma and an MSc from the University of Patras, Greece, and the PhD in Applied Sciences from the Vrije Universiteit Brussel, Belgium. He is Professor of Data and Signal Processing Systems at the Electronic and Electrical Engineering Department of University College London, London, U.K. His research interests are in machine learning and multimedia systems. In his academic work, he has made major contributions to image/vision computing and large-scale multidimensional data analysis. In collaboration with industry, elements of his research in video processing systems have been integrated into commercial products. 